Supporting information

\title{
From Internal Olefins to Linear Amines: Ruthenium-Catalyzed Domino Water-Gas Shift/Hydroaminomethylation Sequence
}

Jie Liu, ${ }^{\dagger}$ Christoph Kubis, ${ }^{\dagger}$ Robert Franke, ${ }^{\ddagger, \S}$ Ralf Jackstell, $^{\dagger}$ and Matthias Beller* ${ }^{\dagger}$

${ }^{\dagger}$ Leibniz-Institut für Katalyse e.V., an der Universität Rostock, Albert-Einstein-Str. 29a, 18059 Rostock, Germany

${ }^{\ddagger}$ Evonik Industries AG, Paul-Baumann-Str. 1, 45772 Marl, Germany

${ }^{\S}$ Lehrstuhl für Theoretische Chemie, 44780 Bochum, Germany

*E-Mail: Matthias.Beller@catalysis.de 


\section{Table of Contents}

General Considerations

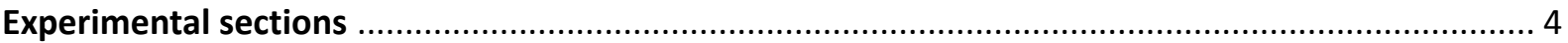

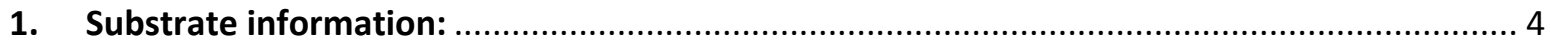

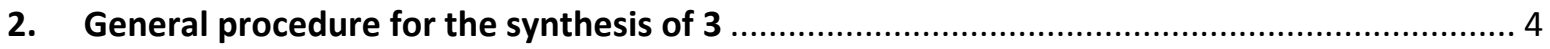

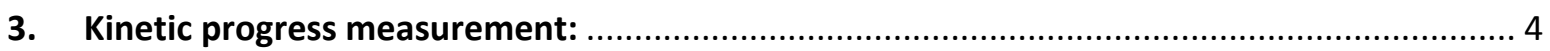

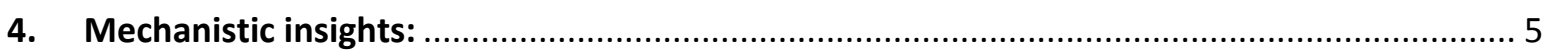

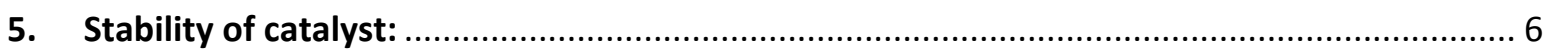

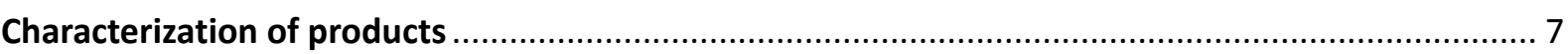

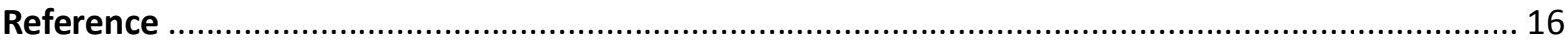

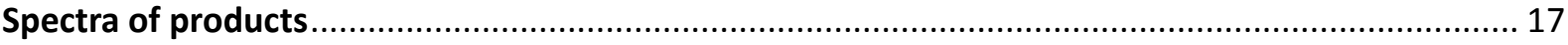




\section{General Considerations}

All commercial reagents were ordered from Alfa Aesar, Aldrich, TCl or Strem. Unless otherwise statement, commercial reagents were used without purification. Air- and moisture-sensitive syntheses were performed under argon atmosphere in heating gun vacuum dried glassware. Analytical data of literature known compounds were in accord with reported data. NMR spectra were recorded on Bruker Avance 300 (300 MHz) NMR spectrometers. Multiplets were assigned as s (singlet), $d$ (doublet), $t$ (triplet), dd (doublet of doublet), $m$ (multiplet) and br. s (broad singlet). All measurements were carried out at room temperature unless otherwise stated. Electron impact (EI) mass spectra were recorded on AMD 402 mass spectrometer (70 eV). High resolution mass spectra (HRMS) were recorded on Agilent 6210 Time-of-Flight LC/MS (Agilent) with electrospray ionization (ESI). The data are given as mass units per charge $(\mathrm{m} / \mathrm{z}$ ) and intensities of signals are given in brackets. For GC analyses, HP 6890 chromatograph with a 29 m HP5 column was used. 


\section{Experimental sections}

\section{Substrate information:}

All the internal olefins and amines are commercial available in Sigma Aldrich, Alfa Aesar, $\mathrm{TCl}$ and Acros. C4 mixture and octene mixture were from Evonik Industry AG.

$\mathrm{Ru}_{3}(\mathrm{CO})_{12}$ was purchase from Sigma Aldrich.

Ligand 2 was synthesized from the known literature. ${ }^{1}$

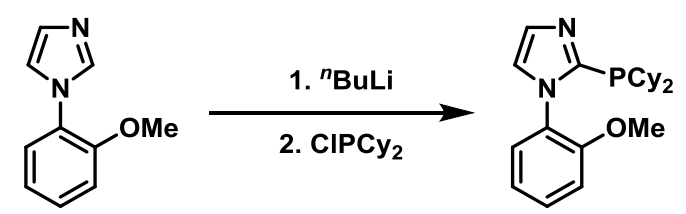

\section{General procedure for the synthesis of 3}

(1) Hydroaminomethylation experiments were carried out in $4 \mathrm{ml}$ reaction vials which were placed in a Parr stainless steel autoclave. In a typical experiment, the reaction vial was charged with $\mathrm{Ru}_{3}(\mathrm{CO})_{12}(0.5 \mathrm{~mol} \%)$, Ligand $2(1.5 \mathrm{~mol} \%)$, internal olefin $(1.3 \mathrm{mmol})$, amine $(1.0 \mathrm{mmol})$, degased water $\left(0.1 \mathrm{ml}\right.$, contain $\left.5 \% \mathrm{Na}_{2} \mathrm{CO}_{3}\right)$ and THF $(1.5 \mathrm{~mL})$ under argon atmosphere. The autoclave was pressurized with 40 bar $\mathrm{CO}$ and heated to $140{ }^{\circ} \mathrm{C}$ for $20 \mathrm{~h}$. After the reaction time, the autoclave was cooled with ice water and the pressure was released. The crude reaction mixture was analyzed by gas chromatography with isooctane or dodecane as internal standard. For isolation, the reaction solvent by vacuum, and the product was purified by column chromatography on silica gel (gradient elution, $\mathrm{n}$ pentane/ethyl acetate $=9: 1$, then ethyl acetate $/ \mathrm{MeOH} 98: 2$ with $0.5 \%$ $\left.\mathrm{Et}_{3} \mathrm{~N}\right)$.

(2) Experiment using crack C4 mixtures: The experiment was carried out in a $25 \mathrm{ml}$ Parr stainless steel autoclave. In a typical experiment, the autoclave was charged with $\mathrm{Ru}_{3}(\mathrm{CO})_{12}(0.2 \mathrm{~mol} \%)$, Ligand 2 (0.6 mol\%), crack C4 mixtures $(0.72 \mathrm{~g})$, piperidine $(10 \mathrm{mmol})$, degased water $(1.0 \mathrm{ml}$, contain $\left.5 \% \mathrm{Na}_{2} \mathrm{CO}_{3}\right)$ and THF $(15.0 \mathrm{~mL})$ under argon atmosphere. The autoclave was pressurized with 50 bar CO and heated to $140{ }^{\circ} \mathrm{C}$ for $72 \mathrm{~h}$. After the reaction time, the autoclave was cooled with ice water and the pressure was released. The regioselectivity was analyzed by gas chromatography with isooctane as internal standard. For isolation, the reaction solvent by vacuum, and the product was purified by column chromatography on silica gel (gradient elution, $\mathrm{n}$ pentane/ethyl acetate $=9: 1$, then ethyl acetate $/ \mathrm{MeOH} 98: 2$ with $\left.0.5 \% \mathrm{Et}_{3} \mathrm{~N}\right)$.

\section{Kinetic progress measurement:}

A $25 \mathrm{ml}$ autoclave was charged under argon atmosphere with $\mathrm{Ru}_{3}(\mathrm{CO})_{12}(0.5$ mol\%), Ligand 2 (1.5 mol\%). Then, 2-octene $(8.0 \mathrm{mmol})$, piperidine $(8.0 \mathrm{mmol})$, isooctane $(0.8 \mathrm{ml}$ as internal), degased water $\left(0.8 \mathrm{ml}\right.$, contain $\left.5 \% \mathrm{Na}_{2} \mathrm{CO}_{3}\right)$ and THF $(12.0 \mathrm{~mL})$, were injected by syringe. Then, the autoclave was pressurized with 40 bar $\mathrm{CO}$ at room temperature. The reaction was heated to $140{ }^{\circ} \mathrm{C}$. Then 
samples were taken from the reaction mixture via a special valve on autoclave in a specific time and analyzed by GC.

\section{Mechanistic insights:}

In order to understand the mechanism for the interaction of this imidazole-type ligand and Ru, some mechanistic studies were performed by using NMR and HRMS:

Based on our previous mechanistic studies, we are thinking about whether $L_{2}$ also has the similar coordination to Ru center. In order to confirm our speculation, the equivalent experiments of $\mathrm{Ru}_{3}(\mathrm{CO})_{12}$ and $\mathrm{L}_{2}$ was done under syn gas ( 40 bar) for 2 hours. The ${ }^{31} \mathrm{P}$ NMR spectra and HRMS data were fast collected from reaction solutions after pressure release at room temperature under $\mathrm{Ar}$ (Scheme S2 and S3). To our delight, the $\mathrm{Ru}(\mathrm{CO})_{3} \mathrm{~L}_{2}$ was confirmed by MS. We attempted to perform an X-ray analysis of $\mathrm{Ru}(\mathrm{CO})_{3} \mathrm{~L}_{2}$, however, the crystallization was not successful because of the instability of this complex. Although currently we do not have very solid evidence to support our experimental results, this interesting finding intrigued us to further investigate the mechanism of this reaction in our future work.

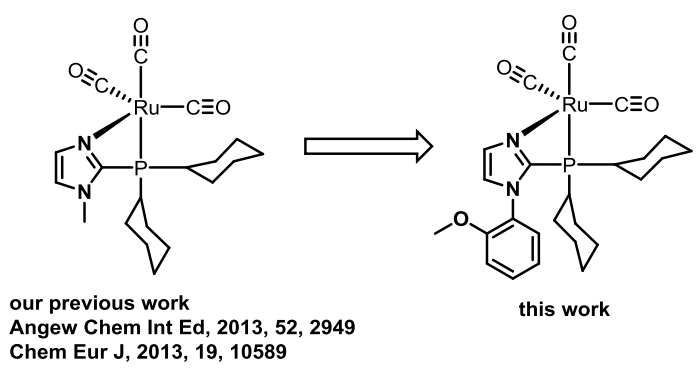

Scheme S1 Putative interaction of two imidazole-type ligands and Ru center.

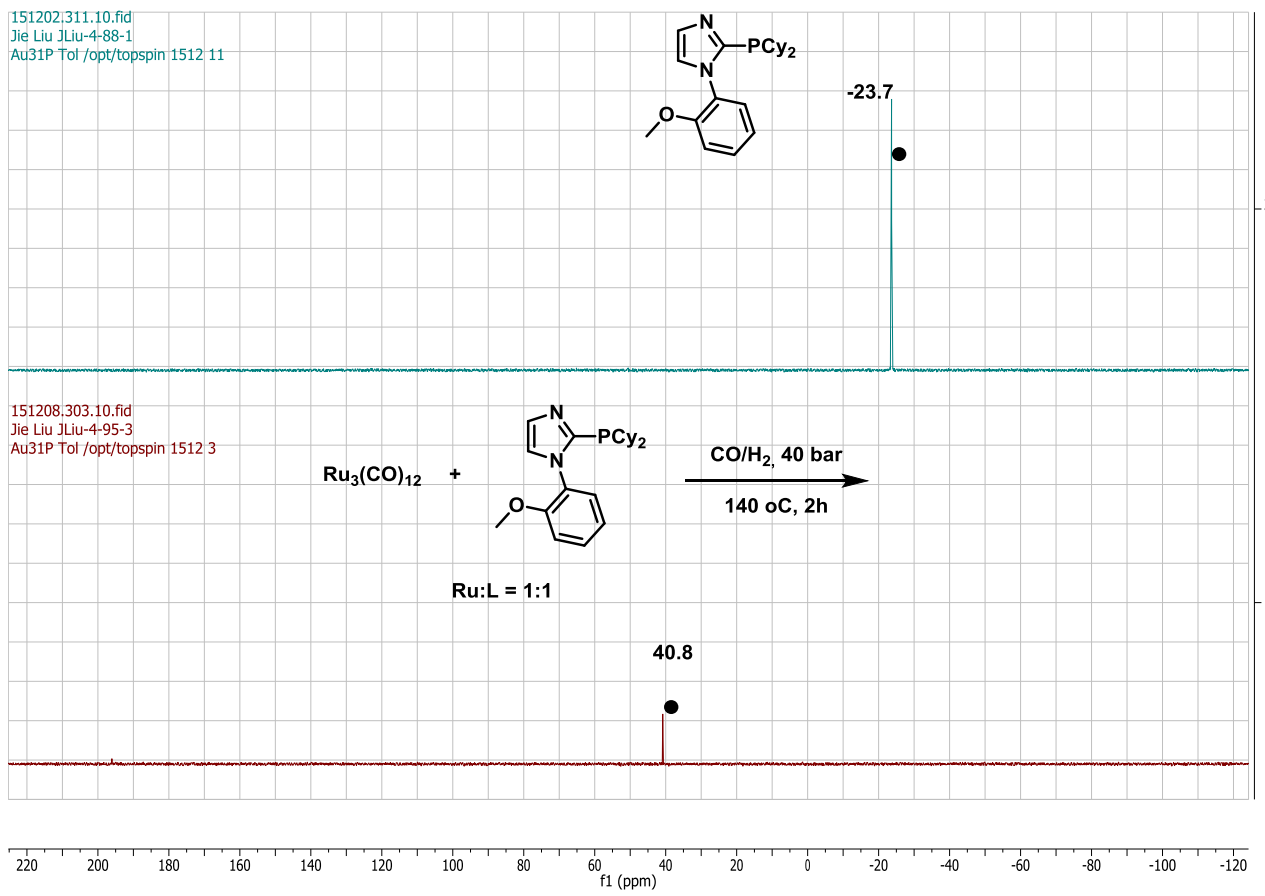




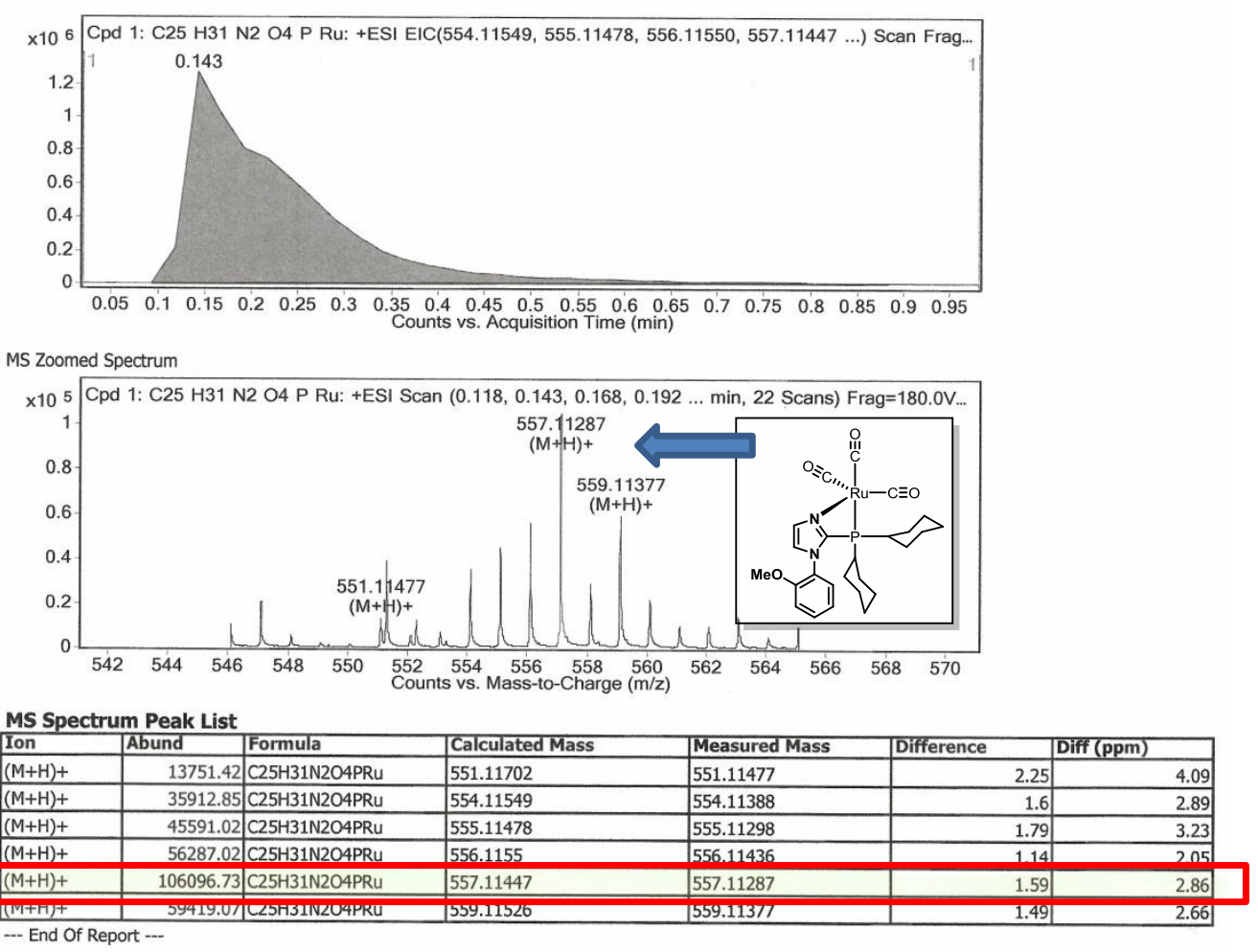

\section{Scheme S3 HRMS spectra}

\section{Stability of catalyst:}

In order to test the stability of the ruthenium catalyst, after $24 \mathrm{~h}$ of reaction, cooling to room temperature, and releasing the pressure, a new portion of substrate and gases was added and the reaction mixture was heated back to $140{ }^{\circ} \mathrm{C}$ (Figure S1). Notably, the reaction took place, however, at a much slower rate. Stopping the reaction after additional $24 \mathrm{~h}$, the products were obtained in $57 \%$ overall yield. This result demonstrated that this catalyst system seems not very stable in the absence of high CO pressure, which is consistent with our mechanistic finding. 


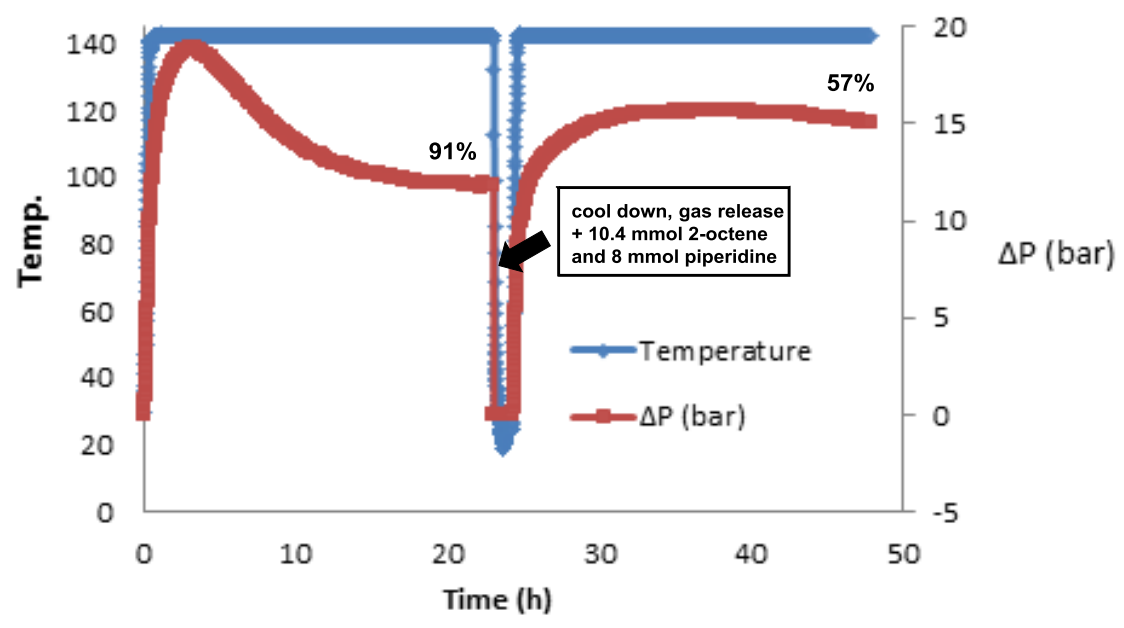

Figure S1: gas consumption for catalyst stability test.

\section{Characterization of products}

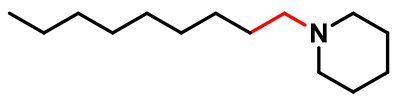

3a

major product, regioselectivity $87: 13$

1-nonylpiperidine ${ }^{2}$

$198.3 \mathrm{mg}$, 94\% yield, colorless liquid. (2-octene as substrate)

${ }^{1} \mathrm{H}$ NMR $\left(300 \mathrm{MHz}, \mathrm{CDCl}_{3}\right)$

反 2.34-2.20 (m, 6H), 1.58-1.49 (m, 4H), 1.47-1.34 (m, 4H), 1.29-1.18 (m, 12H), $0.84(\mathrm{t}, J=6.5 \mathrm{~Hz}, 3 \mathrm{H})$

${ }^{13} \mathrm{CNMR}\left(75 \mathrm{MHz}, \mathrm{CDCl}_{3}\right)$

$\delta 59.98,54.92,32.14,29.89,29.82,29.54,28.05,27.24,26.26,24.78,22.92,14.34$.

HRMS (EI) calculated for $\mathrm{C}_{14} \mathrm{H}_{29} \mathrm{~N}: 211.22945$; found: 211.22916 .

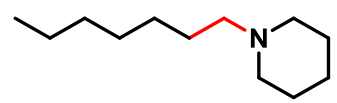

$3 \mathbf{b}$

major product, regioselectivity $89: 11$

1-heptylpiperidine ${ }^{2}$

$155 \mathrm{mg}, 85 \%$ yield, colorless liquid. (2-hexene as substrate) 
${ }^{1} \mathrm{H} \mathrm{NMR}\left(300 \mathrm{MHz}, \mathrm{CDCl}_{3}\right)$

6 2.31-2.27 (m, 3H), 2.22-2.17 (m, 2H), 1.55-1.44 (m, 4H), 1.44-1.33 (m, 4H), 1.24-1.16 (m, 8H), 0.83$0.78(\mathrm{~m}, 3 \mathrm{H})$;

${ }^{13} \mathrm{CNMR}\left(75 \mathrm{MHz}, \mathrm{CDCl}_{3}\right)$

$\delta 59.94,54.89,32.04,29.51,27.97,27.21,26.23,24.75,22.84,14.28$.

HRMS (ESI) calculated for $\left(\mathrm{C}_{12} \mathrm{H}_{25} \mathrm{~N}+\mathrm{H}\right)^{+}:$: 184.20598; found: 184.20619 .

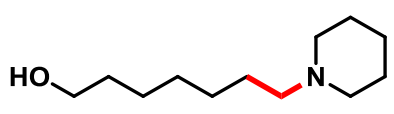

$3 c$

major product, regioselectivity $91: 9$

7-(piperidin-1-yl)heptan-1-ol

$154 \mathrm{mg}, 77 \%$ yield, colorless liquid.

${ }^{1} \mathrm{H}$ NMR $\left(300 \mathrm{MHz}, \mathrm{CDCl}_{3}\right)$

$\delta 3.52(\mathrm{t}, J=6.6 \mathrm{~Hz}, 2 \mathrm{H}), 3.40-3.36(\mathrm{br}, 1 \mathrm{H}), 2.32-2.29(\mathrm{~m}, 4 \mathrm{H}), 2.23-2.18(\mathrm{~m}, 2 \mathrm{H}), 1.54-1.36(\mathrm{~m}, 10 \mathrm{H})$, 1.28-1.23 (m, 6H);

${ }^{13} \mathrm{CNMR}\left(75 \mathrm{MHz}, \mathrm{CDCl}_{3}\right)$

$\delta 62.50,59.77,54.74,32.95,29.57,27.91,26.81,25.95,25.90,24.57$.

HRMS (EI) calculated for $\mathrm{C}_{12} \mathrm{H}_{25} \mathrm{NO}$ : 199.19307; found: 199.19256 .

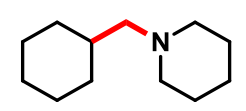

$3 d$

1-(cyclohexylmethyl)piperidine ${ }^{2 a}$

$153.9 \mathrm{mg}, 85 \%$ yield, colorless liquid.

${ }^{1} \mathrm{H}$ NMR $\left(300 \mathrm{MHz}, \mathrm{CDCl}_{3}\right)$

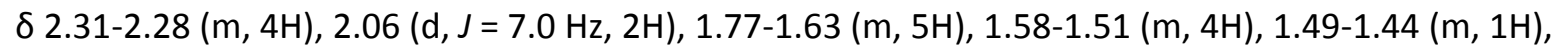
1.43-1.37 (m, 2H), 1.23-1.13 (m, 3H), 0.86-0.81 (m, $2 \mathrm{H})$;

${ }^{13} \mathrm{CNMR}\left(75 \mathrm{MHz}, \mathrm{CDCl}_{3}\right)$

$\delta 66.79,55.36,35.35,32.38,27.05,26.46,26.13,24.77$. 
HRMS (EI) calculated for $\mathrm{C}_{12} \mathrm{H}_{23} \mathrm{~N}$ : 181.18250; found: 181.18258 .

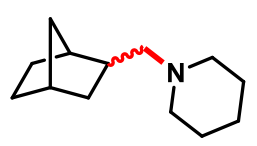

$3 e$

1-(bicyclo[2.2.1]heptan-2-yl)methyl)piperidine, unknown compound.

$150.5 \mathrm{mg}, 78 \%$ yield, colorless liquid.

${ }^{1} \mathrm{H}$ NMR $\left(300 \mathrm{MHz}, \mathrm{CDCl}_{3}\right)$

ठ 2.33-2.31 (m, 4H), 2.23-2.12 (m, 2H), 2.04-2.03 (m, $1 \mathrm{H}), 1.96(\mathrm{dd}, J=12.2,6.9 \mathrm{~Hz}, 1 \mathrm{H}), 1.57-1.50(\mathrm{~m}$, $5 \mathrm{H}), 1.44-1.33(\mathrm{~m}, 5 \mathrm{H}), 1.23-1.19(\mathrm{~m}, 1 \mathrm{H}), 1.12-0.99(\mathrm{~m}, 4 \mathrm{H})$;

${ }^{13} \mathrm{CNMR}\left(75 \mathrm{MHz}, \mathrm{CDCl}_{3}\right)$

$\delta 66.05,55.05,40.48,39.60,37.41,36.62,35.62,30.18,29.08,26.00,24.63$ (exo product)

$63.05,55.02,40.12,37.67,37.21,25.93,24.52,22.94$ (endo product).

HRMS (EI) calculated for $\mathrm{C}_{13} \mathrm{H}_{23} \mathrm{~N}$ : 193.18250; found: 193.18278 .

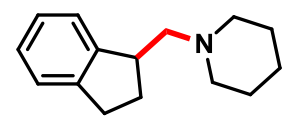

$3 f_{1}$

major product, regioselectivity $87: 13$

2,3-dihydro-1H-inden-1-methyl-piperidine (major product), unknown compound.

$174.3 \mathrm{mg}, 81 \%$ yield, yellow liquid.

${ }^{1} \mathrm{H}$ NMR (300 MHz, $\mathrm{CDCl}_{3}$ )

6 7.54-7.51 (m, $1 \mathrm{H}), 7.38-7.28(\mathrm{~m}, 3 \mathrm{H}), 3.51(\mathrm{p}, J=7.4 \mathrm{~Hz}, 1 \mathrm{H}), 3.14-2.94(\mathrm{~m}, 2 \mathrm{H}), 2.79-2.73(\mathrm{~m}, 1 \mathrm{H})$, 2.68-2.50 (m, 5H), 2.48-2.37 (m, 1H), 2.06-1.94 (m, 1H), 1.83-1.75 (m, 4H), 1.66-1.58 (m, 2H).

${ }^{13} \mathrm{CNMR}\left(75 \mathrm{MHz}, \mathrm{CDCl}_{3}\right)$

$\delta 146.70,144.31,126.54,126.14,124.46,124.44,64.90,55.16,42.53,31.51,31.34,26.31,24.80$.

HRMS (ESI) calculated for $\left(\mathrm{C}_{15} \mathrm{H}_{21} \mathrm{~N}+\mathrm{H}\right)^{+}:$: 216.17468; found: 216.17484 . 


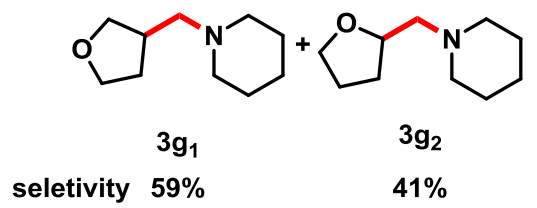

tetrahydrofuran-3-methyl-piperidine; tetrahydrofuran-2- methyl-piperidine $\mathrm{e}^{2 a}$

$120 \mathrm{mg}, 71 \%$ yield, yellow liquid.

${ }^{1} \mathrm{H}$ NMR $\left(300 \mathrm{MHz}, \mathrm{CDCl}_{3}\right)$

б 3.96-3.88 (m, $1 \mathrm{H}), 3.78-3.60(\mathrm{~m}, 5 \mathrm{H}), 3.39-3.34(\mathrm{~m}, 1 \mathrm{H}), 2.38-2.16(\mathrm{~m}, 12 \mathrm{H}), 1.95-1.82(\mathrm{~m}, 2 \mathrm{H}), 1.79-$ $1.68(\mathrm{~m}, 2 \mathrm{H}), 1.51-1.43(\mathrm{~m}, 9 \mathrm{H}), 1.33-1.30(\mathrm{~m}, 5 \mathrm{H})$.

(tetrahydrofuran-3-methyl-piperidine and tetrahydrofuran-2- methyl-piperidine cannot be distinguished in ${ }^{1} \mathrm{H} N M R$, so it is calculated together with a ratio of about 1:1)

${ }^{13} \mathrm{C} \mathrm{NMR}\left(75 \mathrm{MHz}, \mathrm{CDCl}_{3}\right)$

$\delta 76.85,72.61,68.07,67.72,64.19,62.88,55.16,54.90,37.00,31.16,30.58,26.01,25.89,25.43$, $24.48,24.35$.

HRMS (EI) calculated for $\mathrm{C}_{10} \mathrm{H}_{19} \mathrm{ON}$ : 169.14612; found: 169.14576 .

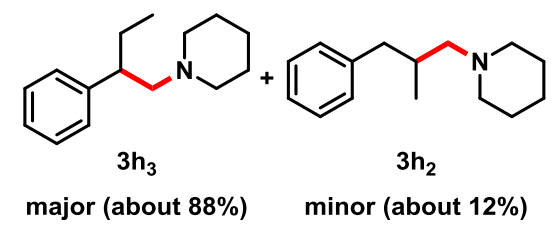

$115 \mathrm{mg}, 53 \%$ yield $\left(3 \mathrm{~h}_{1}+3 \mathrm{~h}_{2}+3 \mathrm{~h}_{3}\right)$, colorless liquid, $1 \mathrm{mmol}$ scale substrate. ${ }^{2 a}$

${ }^{1} \mathrm{H}$ NMR $\left(300 \mathrm{MHz}, \mathrm{CDCl}_{3}\right)$

$\delta$ (major product) 7.23-7.15 (m, 2H), 7.12-7.05 (m, 3H), 2.63-2.56 (m, $1 \mathrm{H}), 2.37-2.20(\mathrm{~m}, 6 \mathrm{H}), 1.51-$ $1.40(\mathrm{~m}, 6 \mathrm{H}), 1.34-1.27(\mathrm{~m}, 2 \mathrm{H}), 0.70-0.65(\mathrm{~m}, 3 \mathrm{H})$;

${ }^{13} \mathrm{CNMR}\left(75 \mathrm{MHz}, \mathrm{CDCl}_{3}\right)$

$\delta$ (major product) $145.25,128.42,128.16,126.17,66.21,55.31,45.78,27.64,26.27,24.74,12.33$;

HRMS (ESI) calculated for $\left(\mathrm{C}_{15} \mathrm{H}_{23} \mathrm{~N}+\mathrm{H}\right)^{+}:$218.19033; found: 218.19051 . 
<smiles>c1ccc(CCCCN2CCCCC2)cc1</smiles>

$3 \mathrm{~h}_{1}$<smiles>CC(Cc1ccccc1)CN1CC[CH+]CC1</smiles>

$3 \mathrm{~h}_{2}$<smiles>CCC(CN1CCCCC1)c1ccccc1</smiles>

$3 h_{3}$

major (about $80 \%$ ) minor (about 13\%)

minor (about 7\%)

${ }^{1} \mathrm{H}$ NMR $\left(300 \mathrm{MHz}, \mathrm{CDCl}_{3}\right)^{2}$

$\delta$ (major product) 7.23-7.08 (m, 5H), $2.55(\mathrm{t}, J=7.3 \mathrm{~Hz}, 2 \mathrm{H}), 2.31-2.20(\mathrm{~m}, 6 \mathrm{H}), 1.57-1.42(\mathrm{~m}, 8 \mathrm{H}), 1.38-$ $1.32(\mathrm{~m}, 2 \mathrm{H})$;

${ }^{13} \mathrm{C} \mathrm{NMR}\left(75 \mathrm{MHz}, \mathrm{CDCl}_{3}\right)$

$\delta$ (major product) 142.81, 128.64, 128.49, 125.88, 59.69, 54.92, 36.15, 29.88, 26.89, 26.24, 24.75.

HRMS (ESI) calculated for $\mathrm{C}_{15} \mathrm{H}_{23} \mathrm{~N}$ : 218.19033; found: 218.19051.

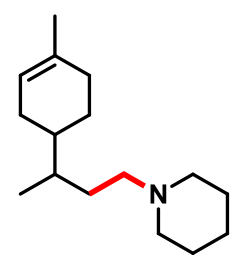

$3 \mathbf{i}$

major product, regioselectivity: 99:1

1-(3-(4-methylcyclohex-3-en-1-yl)butyl)piperidine, unknown compound.

$135.7 \mathrm{mg}, 58 \%$ yield, colorless liquid.

${ }^{1} \mathrm{H}$ NMR $\left(300 \mathrm{MHz}, \mathrm{CDCl}_{3}\right)$

d 5.31-5.29 (m, 1H), 2.32-2.15 (m, 6H), 1.93-1.82 (m, 3H), 1.72-1.48 (m, 10H), 1.39-1.19 (m, 6H), 0.83$0.77(\mathrm{~m}, 3 \mathrm{H})$.

${ }^{13} \mathrm{CNMR}\left(75 \mathrm{MHz}, \mathrm{CDCl}_{3}\right)$

$\delta 133.99,121.21,58.28,54.96,38.92,38.66,36.20,36.06,31.42,31.18,31.13,31.04,29.50,27.76$, $27.17,26.19,25.68,24.71,23.62,16.64,16.24$.

HRMS (ESI) calculated for $\left(\mathrm{C}_{16} \mathrm{H}_{29} \mathrm{~N}+\mathrm{H}\right)^{+}: 236.23728$; found: 236.23750 .

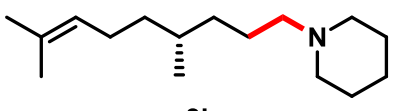

3j

major product, regioselectivity: 99:1

(R)-1-(4,8-dimethylnon-7-en-1-yl)piperidine ${ }^{2 b}$ 
$213.5 \mathrm{mg}, 90 \%$ yield, colorless liquid.

${ }^{1} \mathrm{H}$ NMR $\left(300 \mathrm{MHz}, \mathrm{CDCl}_{3}\right)$

ס 5.04-4.97 (m, $1 \mathrm{H}), 2.29-2.13(\mathrm{~m}, 6 \mathrm{H}), 1.95-1.80(\mathrm{~m}, 2 \mathrm{H}), 1.60-1.59(\mathrm{~m}, 3 \mathrm{H}), 1.51-1.46(\mathrm{~m}, 7 \mathrm{H}), 1.45-$ $1.28(\mathrm{~m}, 5 \mathrm{H}), 1.27-1.16(\mathrm{~m}, 2 \mathrm{H}), 1.09-0.99(\mathrm{~m}, 2 \mathrm{H}), 0.79(\mathrm{~d}, J=6.4 \mathrm{~Hz}, 3 \mathrm{H})$

${ }^{13} \mathrm{CNMR}\left(75 \mathrm{MHz}, \mathrm{CDCl}_{3}\right)$

$\delta$ 130.96, 125.11, 60.16, 54.84, 37.19, 35.02, 32.54, 26.17, 25.84, 25.69, 24.69, 24.59, 19.73, 17.74.

HRMS (EI) calculated for $\mathrm{C}_{16} \mathrm{H}_{31} \mathrm{~N}: 237.24510$; found: 237.24483 .

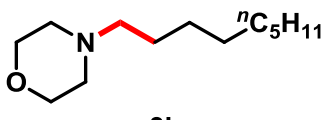

3k

major product, regioselectivity: $85: 15$

4-nonylmorpholine ${ }^{2}$

$138.6 \mathrm{mg}, 65 \%$ yield, colorless liquid.

${ }^{1} \mathrm{H}$ NMR $\left(300 \mathrm{MHz}, \mathrm{CDCl}_{3}\right)$

反 3.62-3.59 (m, $4 \mathrm{H}), 2.33-2.30(\mathrm{~m}, 4 \mathrm{H}), 2.23-2.18(\mathrm{~m}, 2 \mathrm{H}), 1.40-1.33(\mathrm{~m}, 2 \mathrm{H}), 1.18-1.15(\mathrm{~m}, 12 \mathrm{H}), 0.80-$

$0.75(\mathrm{~m}, 3 \mathrm{H})$.

${ }^{13} \mathrm{C} \mathrm{NMR}\left(75 \mathrm{MHz}, \mathrm{CDCl}_{3}\right)$

$\delta 67.08,59.35,53.93,32.00,29.70,29.67,29.40,27.64,26.70,22.78,14.20$.

HRMS (EI) calculated for $\mathrm{C}_{13} \mathrm{H}_{27} \mathrm{NO}$ : 213.20872; found: 213.20864

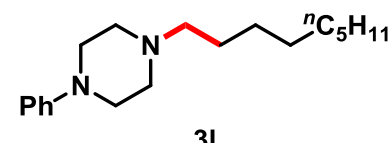

major product, regioselectivity: $84: 16$

1-nonyl-4-phenylpiperazine ${ }^{2}$

$187.1 \mathrm{mg}, 65 \%$ yield, colorless liquid.

${ }^{1} \mathrm{H} \mathrm{NMR}\left(300 \mathrm{MHz}, \mathrm{CDCl}_{3}\right)$

反 7.19-7.14 (m, 2H), 6.86-6.82 (m, 2H), 6.78-6.73 (m, 1H), 3.13-3.10 (m, 4H), 2.52-2.49 (m, 4H), 2.32$2.26(\mathrm{~m}, 2 \mathrm{H}), 1.47-1.42(\mathrm{~m}, 2 \mathrm{H}), 1.25-1.16(\mathrm{~m}, 12 \mathrm{H}), 0.83-0.78(\mathrm{~m}, 3 \mathrm{H})$. 
${ }^{13} \mathrm{CNMR}\left(75 \mathrm{MHz}, \mathrm{CDCl}_{3}\right)$

$\delta 151.57,129.25,119.77,116.18,59.08,53.53,49.33,32.11,29.83,29.79,29.51,27.85,27.15,22.90$, 14.35 .

HRMS (EI) calculated for $\mathrm{C}_{19} \mathrm{H}_{32} \mathrm{~N}_{2}: 288.25600$; found: 288.25580 .

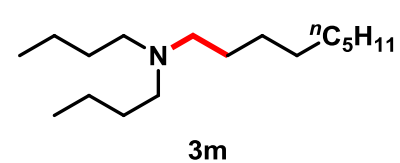

major product, regioselectivity: $86: 14$

$N, N$-dibutylnonan-1-amine, unknown compound.

$221.9 \mathrm{mg}, 87 \%$ yield, colorless liquid.

${ }^{1} \mathrm{H}$ NMR $\left(300 \mathrm{MHz}, \mathrm{CDCl}_{3}\right)$

ठ 2.40-2.34 (m, 6H), 1.45-1.38 (m, 6H), 1.37-1.22 (m, 16H), 0.93-0.83 (m, 9H)

${ }^{13} \mathrm{CNMR}\left(75 \mathrm{MHz}, \mathrm{CDCl}_{3}\right)$

$\delta 54.58,54.26,32.20,29.96,29.93,29.61,29.57,27.97,27.38,22.98,21.11,14.40$.

HRMS (ESI) calculated for $\left(\mathrm{C}_{17} \mathrm{H}_{37} \mathrm{~N}+\mathrm{H}\right)^{+}:$:256.29988; found: 256.30015.

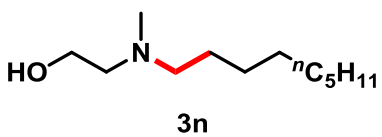

major product, regioselectivity: 75:25

2-(methyl(nonyl)amino)ethan-1-ol ${ }^{2}$

$154.8 \mathrm{mg}, 77 \%$ yield, colorless liquid.

${ }^{1} \mathrm{H}$ NMR $\left(300 \mathrm{MHz}, \mathrm{CDCl}_{3}\right)$

$\delta 3.50(\mathrm{t}, J=5.5 \mathrm{~Hz}, 2 \mathrm{H}), 2.96-2.82(\mathrm{~m}, 1 \mathrm{H},[\mathrm{OH}]), 2.43(\mathrm{t}, J=5.5 \mathrm{~Hz}, 2 \mathrm{H}), 2.33-2.28(\mathrm{~m}, 2 \mathrm{H}), 2.16(\mathrm{~s}$, $3 \mathrm{H}), 1.41-1.36(\mathrm{~m}, 2 \mathrm{H}), 1.23-1.18(\mathrm{~m}, 12 \mathrm{H}), 0.82-0.78(\mathrm{~m}, 3 \mathrm{H})$;

${ }^{13} \mathrm{CNMR}\left(75 \mathrm{MHz}, \mathrm{CDCl}_{3}\right)$

$\delta$ (major product) 59.12, 58.64, 58.06, 41.84, 32.02, 32.00, 29.71, 29.41, 27.52, 27.33, 22.78, 14.20;

$\delta$ (minor product) 59.99, 59.60, 58.87, 52.18, 42.20, 36.01, 35.19, 31.16, 30.00, 29.77, 27.01, 18.28. 
HRMS (EI) calculated for $\mathrm{C}_{12} \mathrm{H}_{27} \mathrm{NO}$ : 201.20872; found: 201.20847.

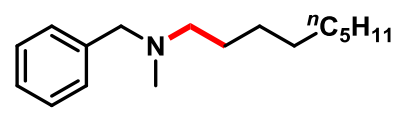

30

major product, regioselectivity: $84: 16$

$\mathrm{N}$-benzyl-N-methylnonan-1-amine, unknown compound.

$155.6 \mathrm{mg}, 63 \%$ yield, colorless liquid.

${ }^{1} \mathrm{H}$ NMR $\left(300 \mathrm{MHz}, \mathrm{CDCl}_{3}\right)$

$\delta$ 7.24-7.12 (m, 5H), $3.38(\mathrm{~s}, 2 \mathrm{H}), 2.29-2.24(\mathrm{~m}, 2 \mathrm{H}), 2.09(\mathrm{~s}, 3 \mathrm{H}), 1.45-1.40(\mathrm{~m}, 2 \mathrm{H}), 1.25-1.18(\mathrm{~m}, 12 \mathrm{H})$, $0.82-0.78(\mathrm{~m}, 3 \mathrm{H})$

${ }^{13} \mathrm{C} \mathrm{NMR}\left(75 \mathrm{MHz}, \mathrm{CDCl}_{3}\right)$

$139.58,129.28,128.39,127.06,62.62,57.89,42.51,32.18,29.89,29.59,27.74,27.71,22.96,14.38$.

HRMS (EI) calculated for $\mathrm{C}_{17} \mathrm{H}_{29} \mathrm{~N}: 247.22945$; found: 247.22951 .

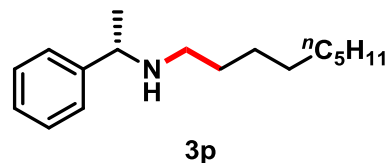

major product, regioselectivity: 85:15

(S)-N-(1-phenylethyl)nonan-1-amine ${ }^{2 a}$

$86.4 \mathrm{mg}, 35 \%$ yield, colorless liquid.

${ }^{1} \mathrm{H}$ NMR $\left(300 \mathrm{MHz}, \mathrm{CDCl}_{3}\right)$

6 7.24-7.11 (m, 5H), $3.67(\mathrm{q}, J=6.6 \mathrm{~Hz}, 1 \mathrm{H}), 2.45-2.28(\mathrm{~m}, 2 \mathrm{H}), 1.40-1.34(\mathrm{~m}, 3 \mathrm{H}), 1.27(\mathrm{~d}, J=6.6 \mathrm{~Hz}$, $3 \mathrm{H}), 1.17-1.16(\mathrm{~m}, 12 \mathrm{H}), 0.82-0.77(\mathrm{~m}, 3 \mathrm{H})$.

${ }^{13} \mathrm{C} \mathrm{NMR}\left(75 \mathrm{MHz}, \mathrm{CDCl}_{3}\right)$

$\delta 146.14,128.61,127.03,126.78,58.66,48.16,32.13,30.55,29.81,29.52,27.62,24.59,22.92,14.35$.

HRMS (ESI) calculated for $\left(\mathrm{C}_{17} \mathrm{H}_{29} \mathrm{~N}+\mathrm{H}\right)^{+}:$248.23728; found: 248.23739 . 


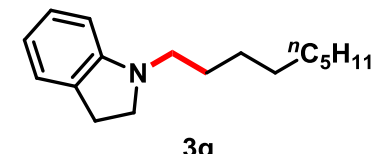

$3 q$

major product, regioselectivity: $86: 14$

1-nonylindoline ${ }^{2}$

$44.1 \mathrm{mg}, 18 \%$ yield, colorless liquid.

${ }^{1} \mathrm{H} \mathrm{NMR}\left(300 \mathrm{MHz}, \mathrm{CDCl}_{3}\right)$

反 7.10-7.05 (m, 2H), 6.67-6.63 (m, 1H), 6.50-6.47 (m, 1H), $3.36(\mathrm{t}, J=8.3 \mathrm{~Hz}, 2 \mathrm{H}),[3.09-3.06(\mathrm{~m}, 1.72$ $\mathrm{H}$, normal product), 2.92-2.82 (m, $0.24 \mathrm{H}$, iso-product)], $2.98(\mathrm{t}, J=8.3 \mathrm{~Hz}, 2 \mathrm{H}), 1.65-1.60(\mathrm{~m}, 2 \mathrm{H})$, $1.42-1.30(\mathrm{~m}, 12 \mathrm{H}), 0.94-0.90(\mathrm{~m}, 3 \mathrm{H})$;

${ }^{13} \mathrm{CNMR}\left(75 \mathrm{MHz}, \mathrm{CDCl}_{3}\right)$

$\delta$ 153.04, 130.25, 127.51, 124.58, 117.45, 107.09, 53.32, 49.59, 32.18, 29.89, 29.83, 29.59, 28.85, $27.63,27.57,22.96,14.40$.

HRMS (EI) calculated for $\mathrm{C}_{17} \mathrm{H}_{27} \mathrm{~N}: 245.21380$; found: 245.21375 .

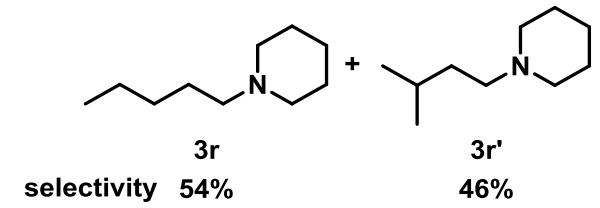

1-pentylpiperidine; iso pentylpiperidine ${ }^{3}$

Product distribution: $44 \%$ yield of 1-pentylpiperidine, $37 \%$ yield of 1-isopentylpiperidine, colorless liquid, determined by GC.

${ }^{1} \mathrm{H} \mathrm{NMR}\left(300 \mathrm{MHz}, \mathrm{CDCl}_{3}\right)$

ठ 2.31-2.17 (m, 12H), 1.56-1.48 (m, 8H), 1.47-1.19 (m, 13H), 0.85-0.80 (m, 9H) (1-pentylpiperidine and 1-isopentylpiperidine cannot be distinguished in ${ }^{1} \mathrm{H}$ NMR, so it is calculated together with a ratio of about 1:1)

${ }^{13} \mathrm{C} \mathrm{NMR}\left(75 \mathrm{MHz}, \mathrm{CDCl}_{3}\right)$

$\delta$ 1-pentylpiperidine: $59.89,54.88,30.19,26.88,26.22,24.74,22.95,14.23$;

$\delta$ 1-isopentylpiperidine: 58.09, 54.94, 36.20, 27.11, 26.24, 22.86.

HRMS (ESI) calculated for $\left(\mathrm{C}_{10} \mathrm{H}_{21} \mathrm{~N}+\mathrm{H}\right)^{+}:$: 156.17468; found: 156.17474 . 


\section{Reference}

1. Wu, L.; Fleischer, I.; Jackstell, R.; Profir, I.; Franke, R.; Beller, M., Ruthenium-Catalyzed Hydroformylation/Reduction of Olefins to Alcohols: Extending the Scope to Internal Alkenes. J. Am. Chem. Soc. 2013, 135, 14306-14312.

2. (a) Wu, L.; Fleischer, I.; Jackstell, R.; Beller, M., Efficient and Regioselective Rutheniumcatalyzed Hydro-aminomethylation of Olefins. J. Am. Chem. Soc. 2013, 135, 3989-3996; (b) Gülak, S.; Wu, L.; Liu, Q.; Franke, R.; Jackstell, R.; Beller, M., Phosphine- and Hydrogen-Free: Highly Regioselective Ruthenium-Catalyzed Hydroaminomethylation of Olefins. Angew. Chem. Int. Ed. 2014, 53, 7320-7323.

3. Seayad, A.; Ahmed, M.; Klein, H.; Jackstell, R.; Gross, T.; Beller, M., Internal Olefins to Linear Amines. Science 2002, 297, 1676-1678. 


\section{Spectra of products}

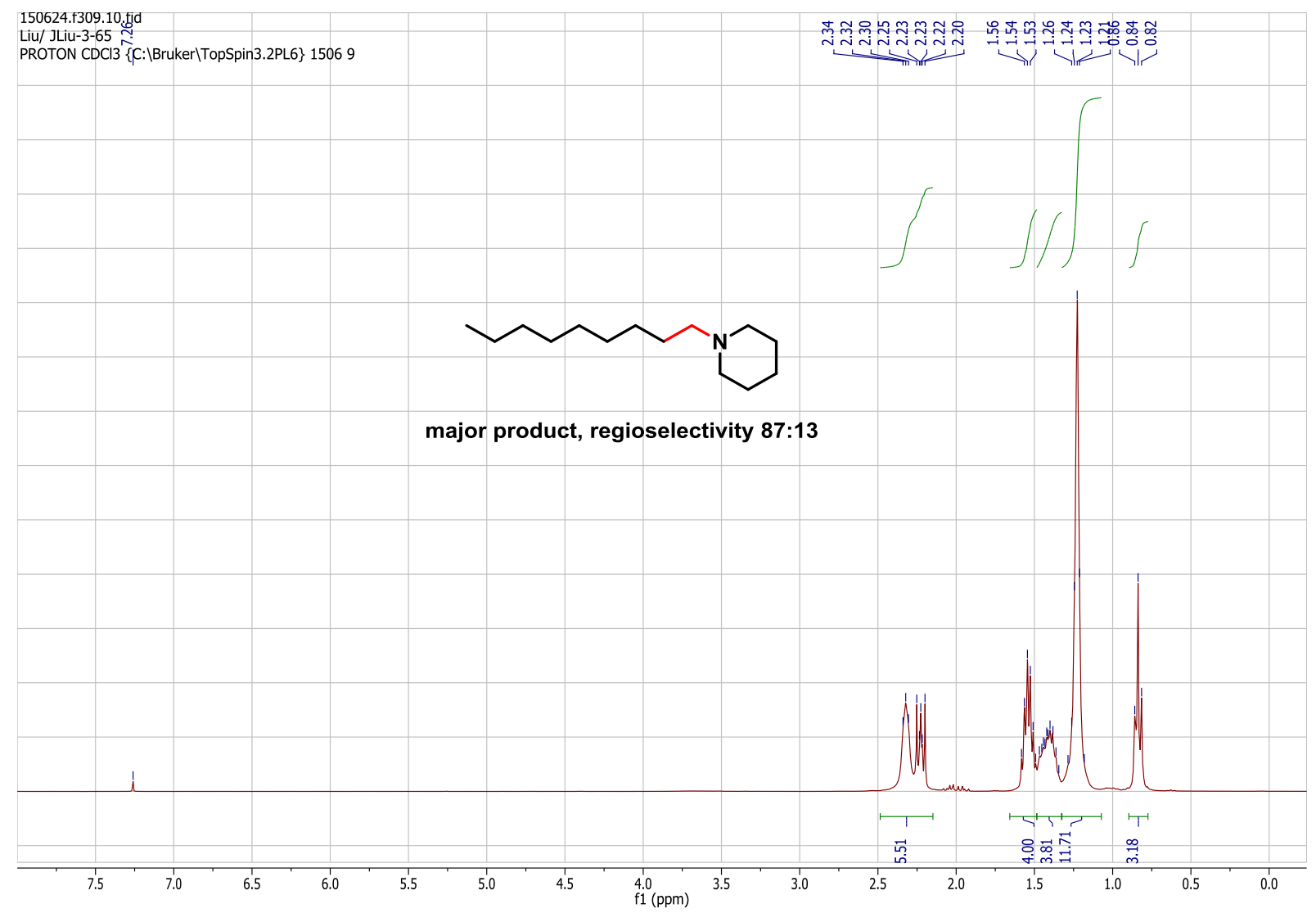

150624.f309.11.fid

C13CPD CDCl3 \{C:|Bruker|TopSpin3.2PL6\} 15069

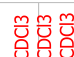

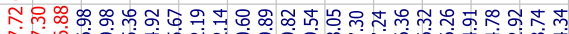

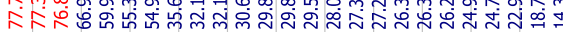

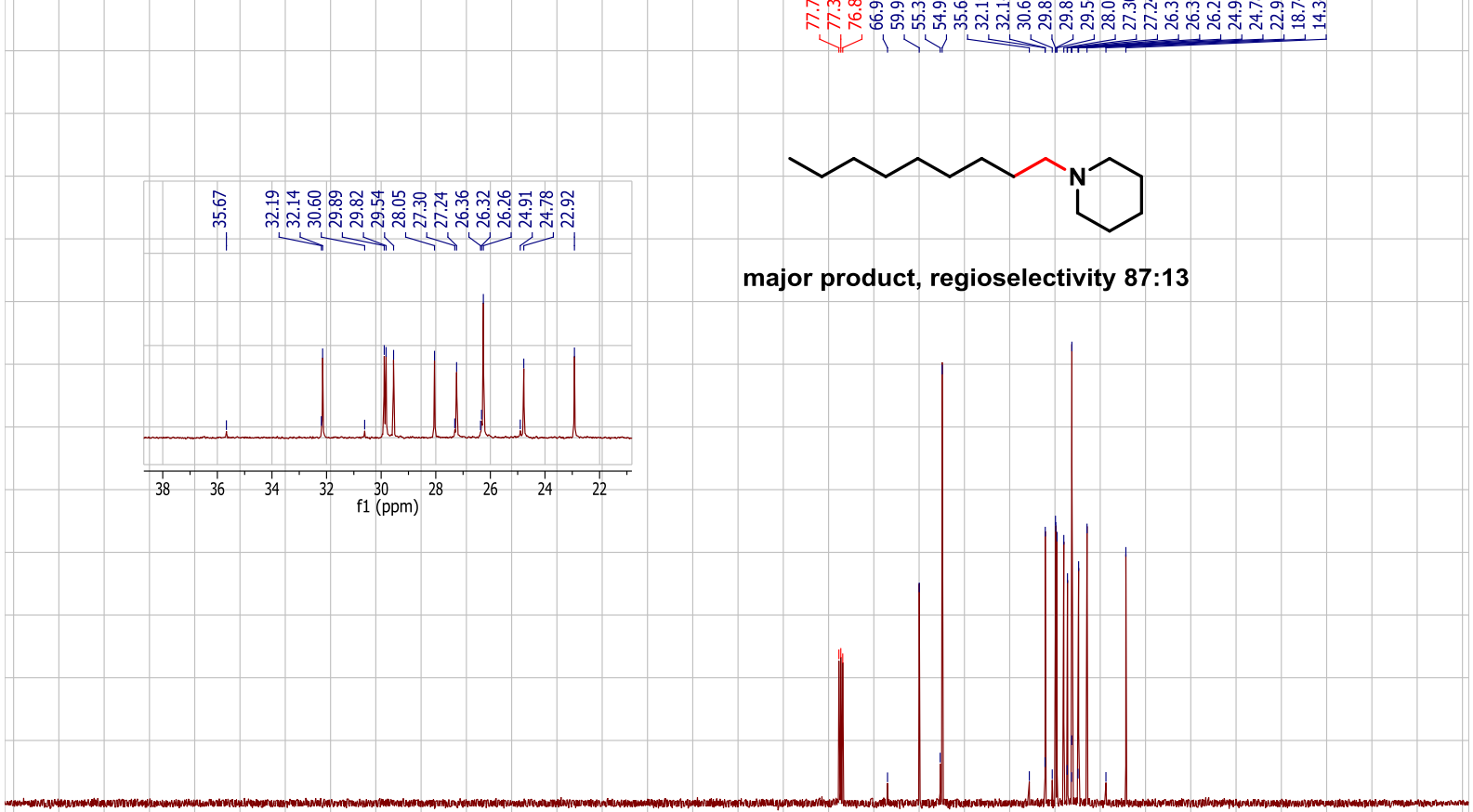

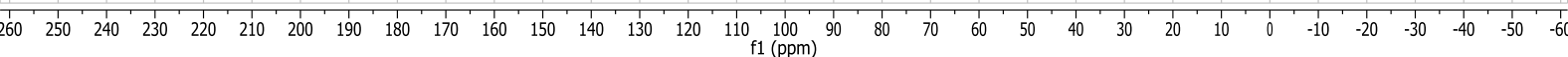



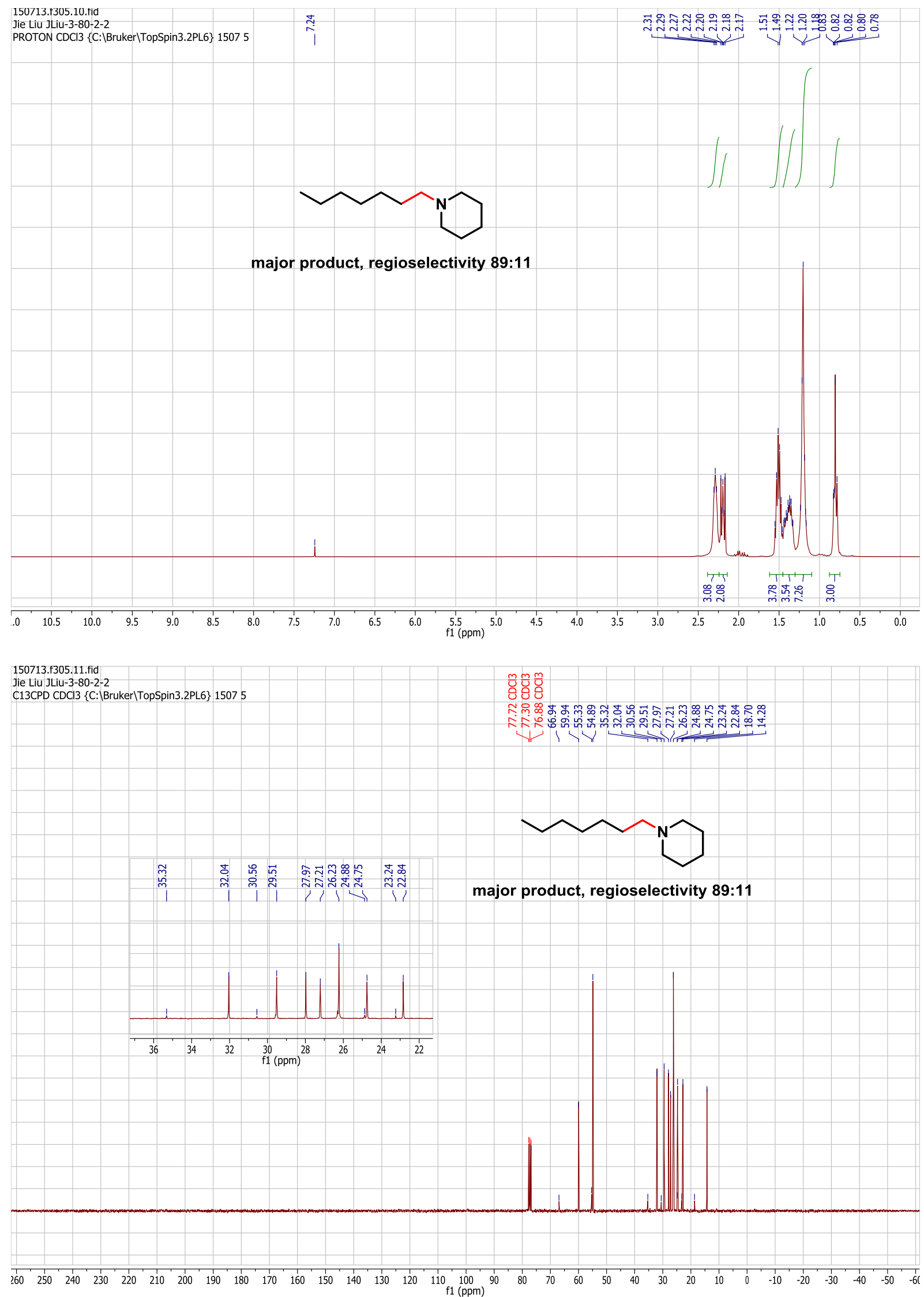


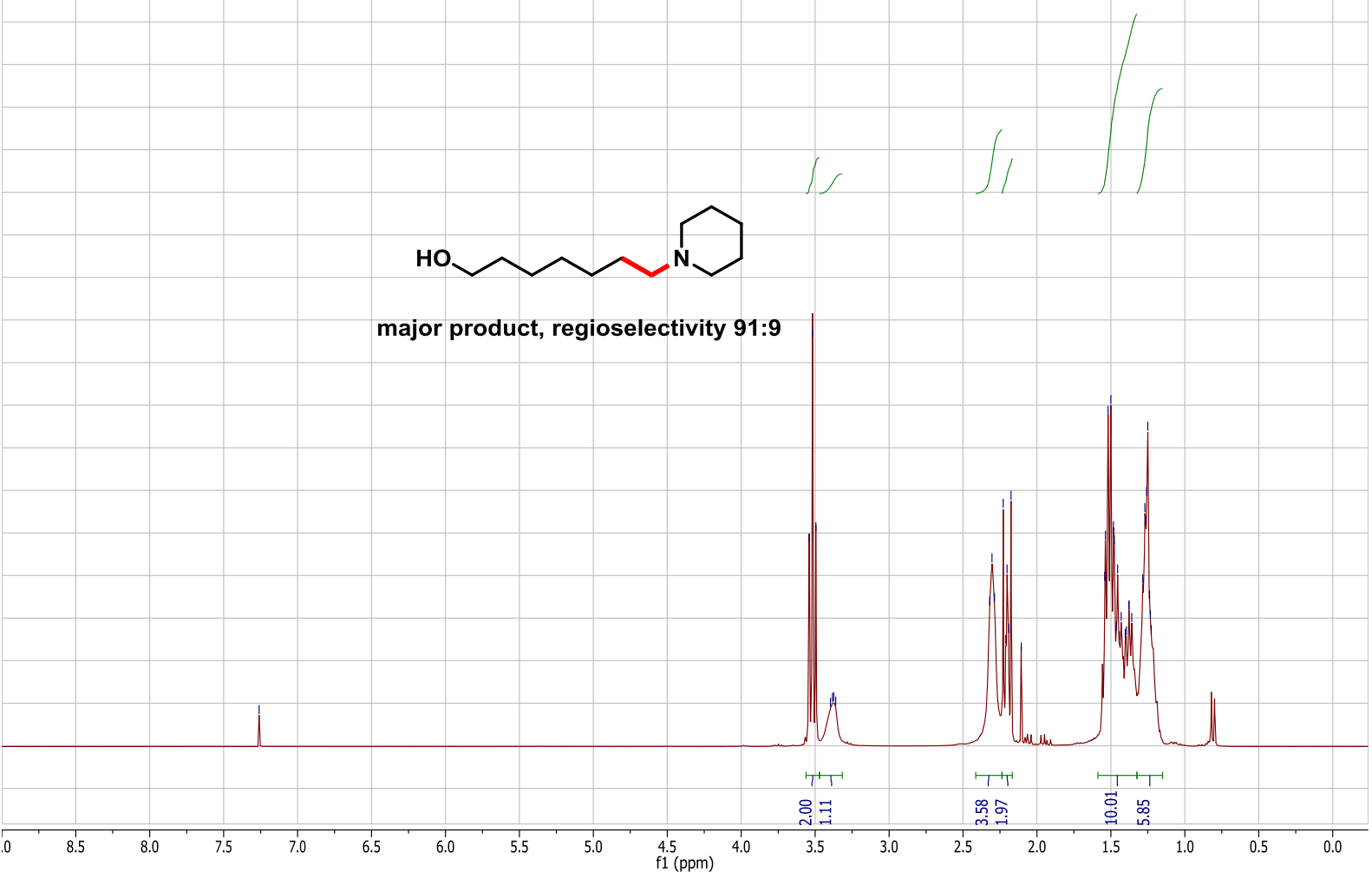

150724.347.11. fid
Jie Liu JLiu-3-104-7

Jie Liu JLiu-3-104-7
Au13C CDCl3 /opt/topspin 150747

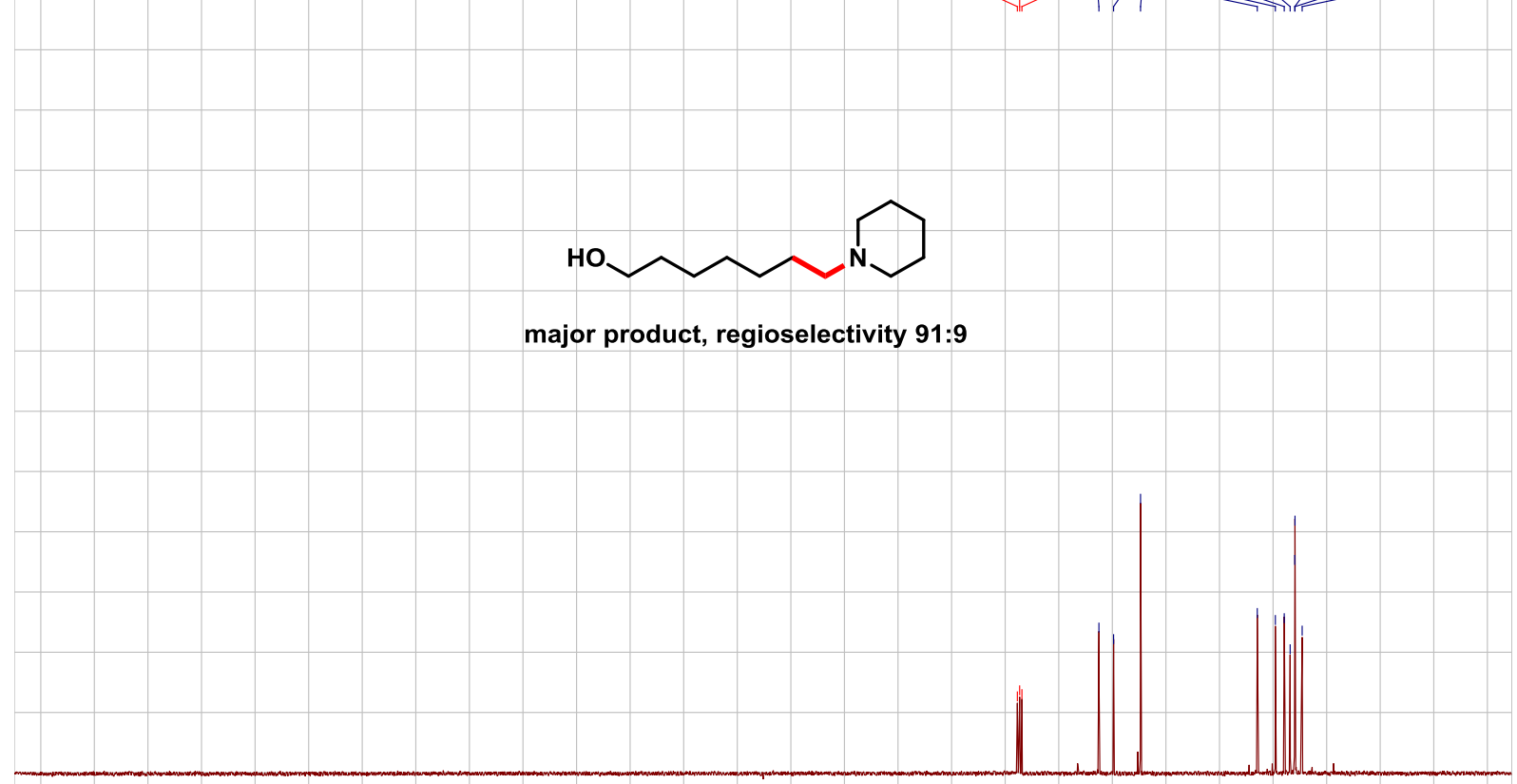




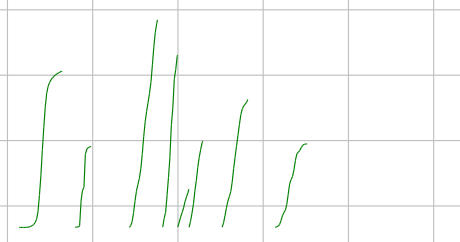

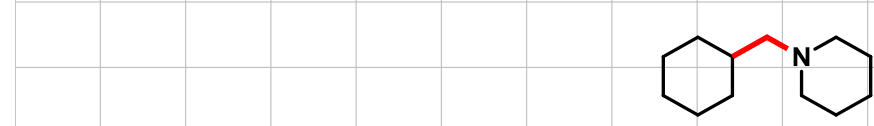

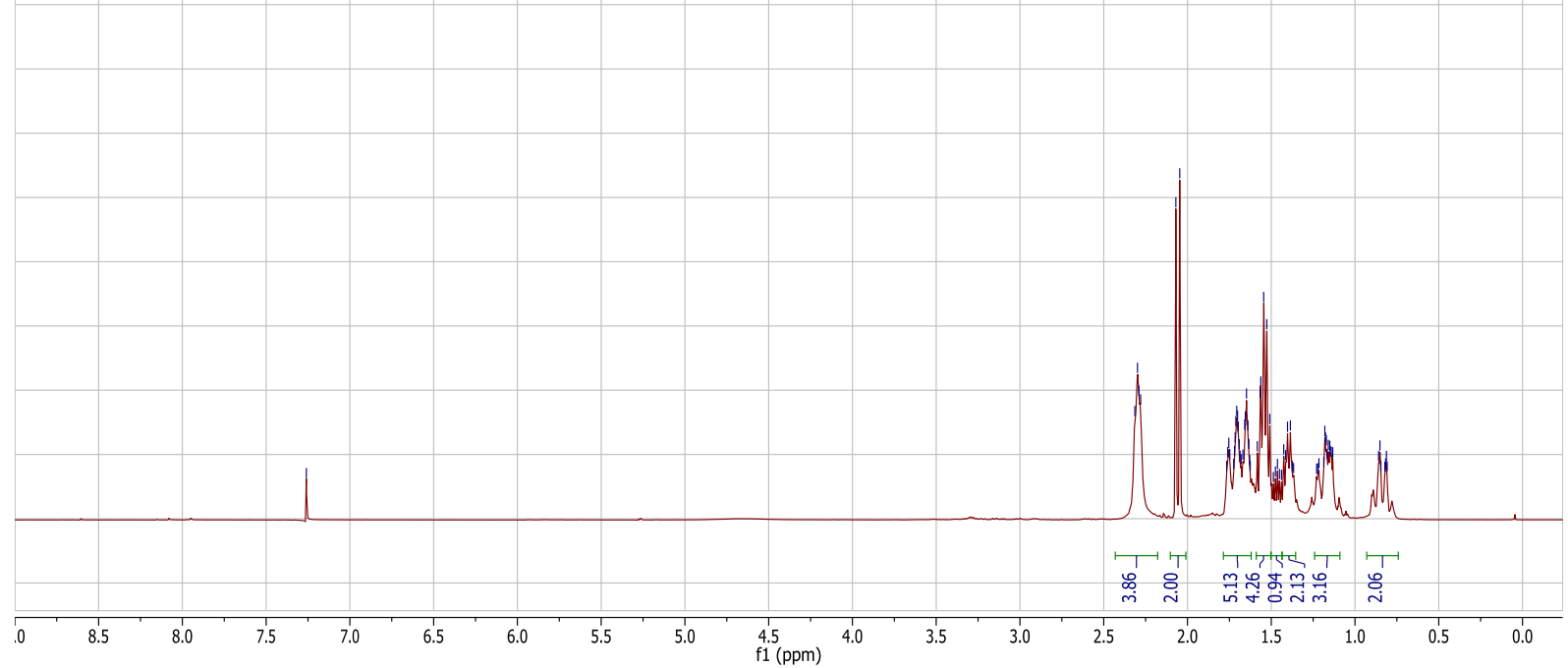

150629.f320.11.fid

C13CPD CDCl3 \{C:|Bruker|TopSpin3.2PL6\} 150620

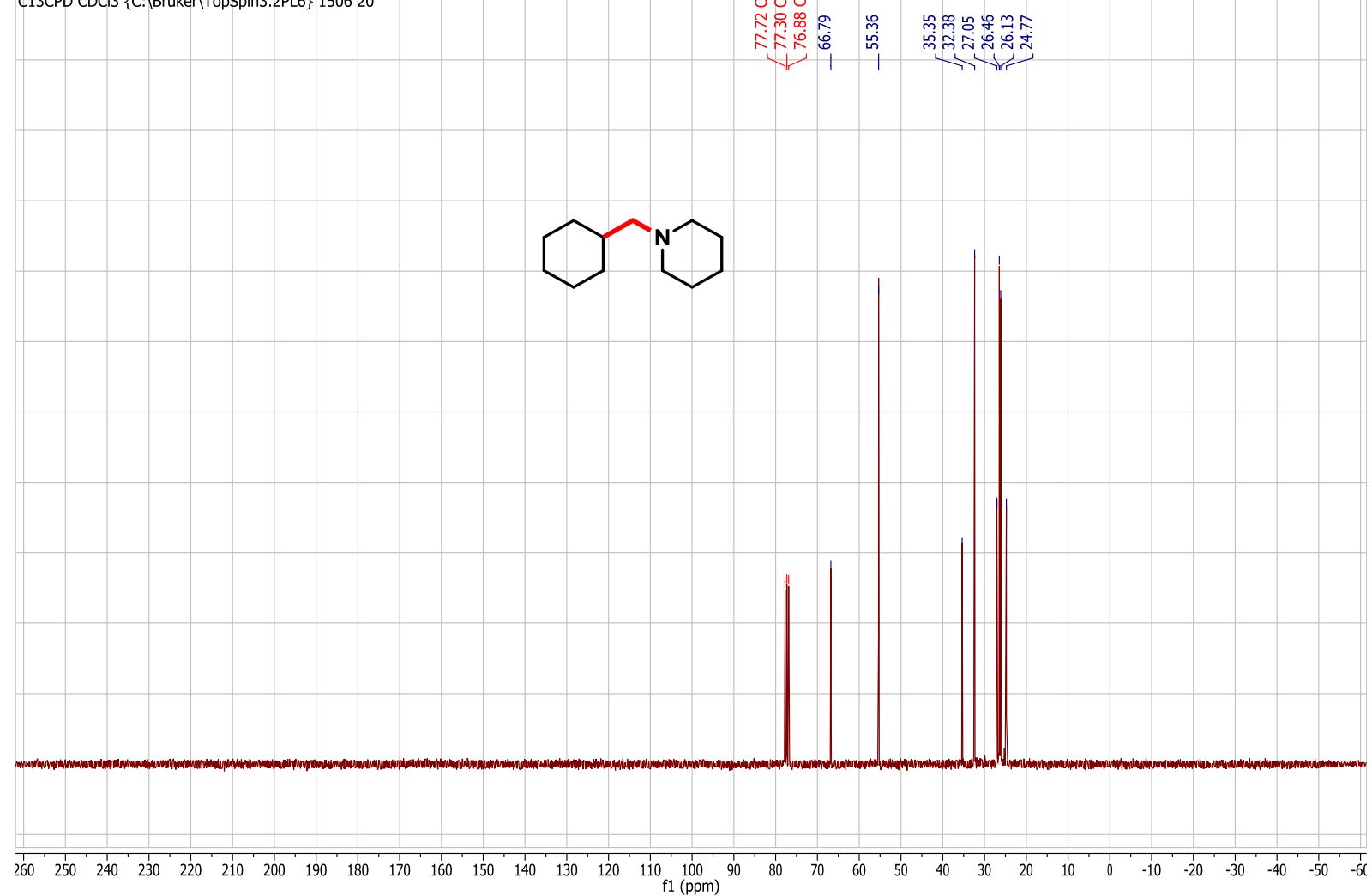




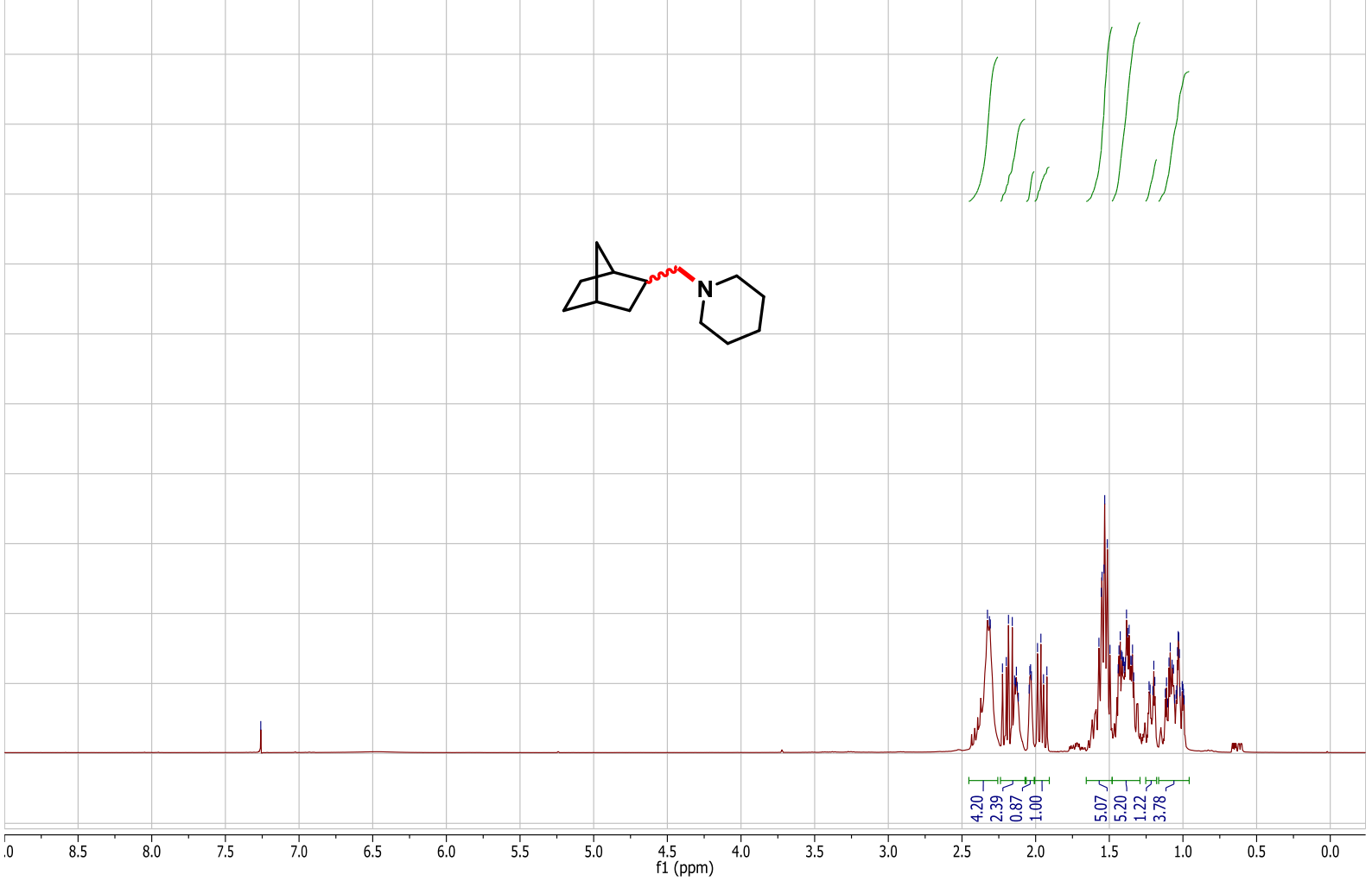

150716.f306.11.fid
Jie Liu JLiu-3-80-6-2

C13CPD CDCI3 \{C:|Bruker|TopSpin3.2PL6\} 15076

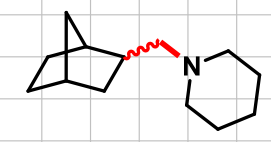

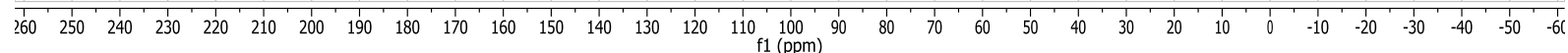




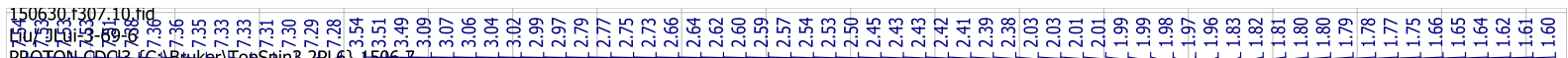
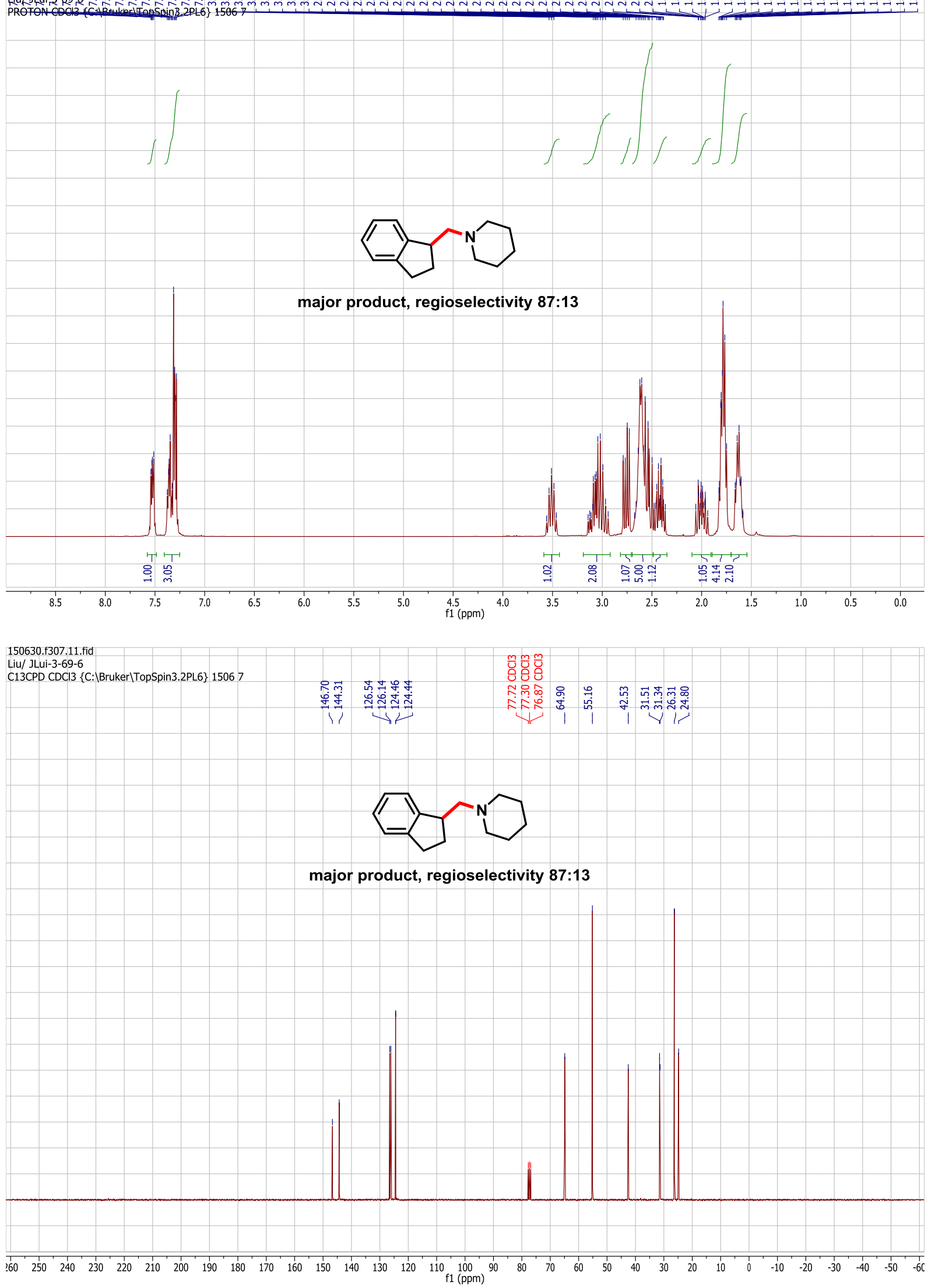

S22 


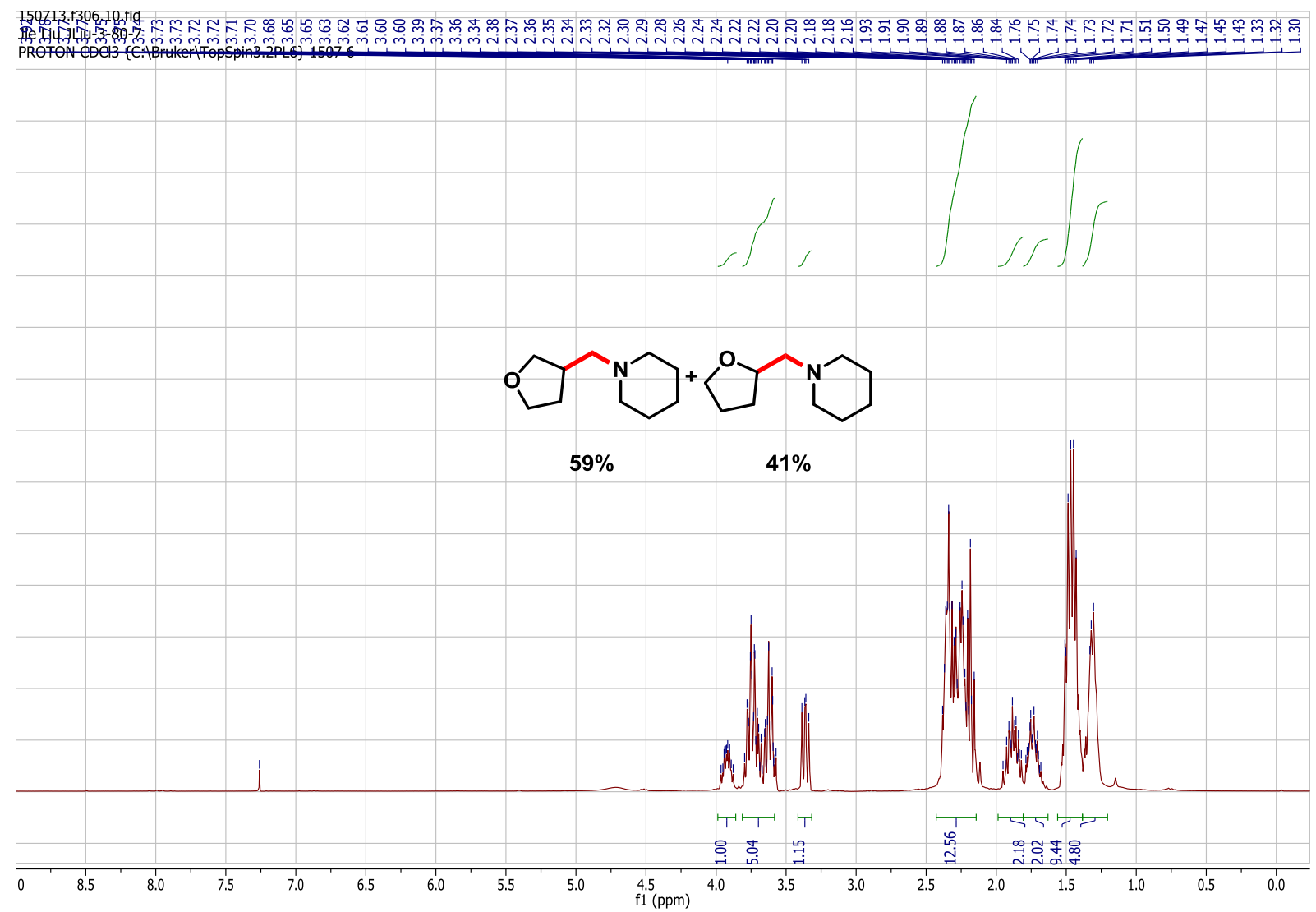

150713.f306.11.fid
Jie Liu JLiu-3-80-7

C13CPD CDCI3 \{C:|Bruker|TopSpin3.2PL6\} 15076

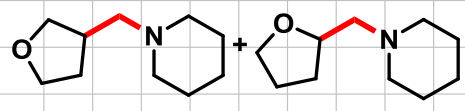

$59 \%$

$41 \%$

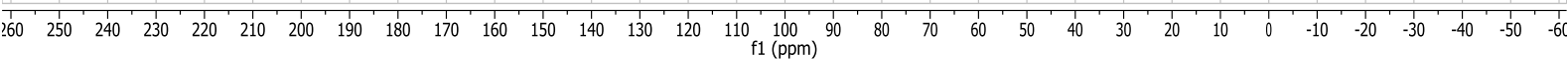




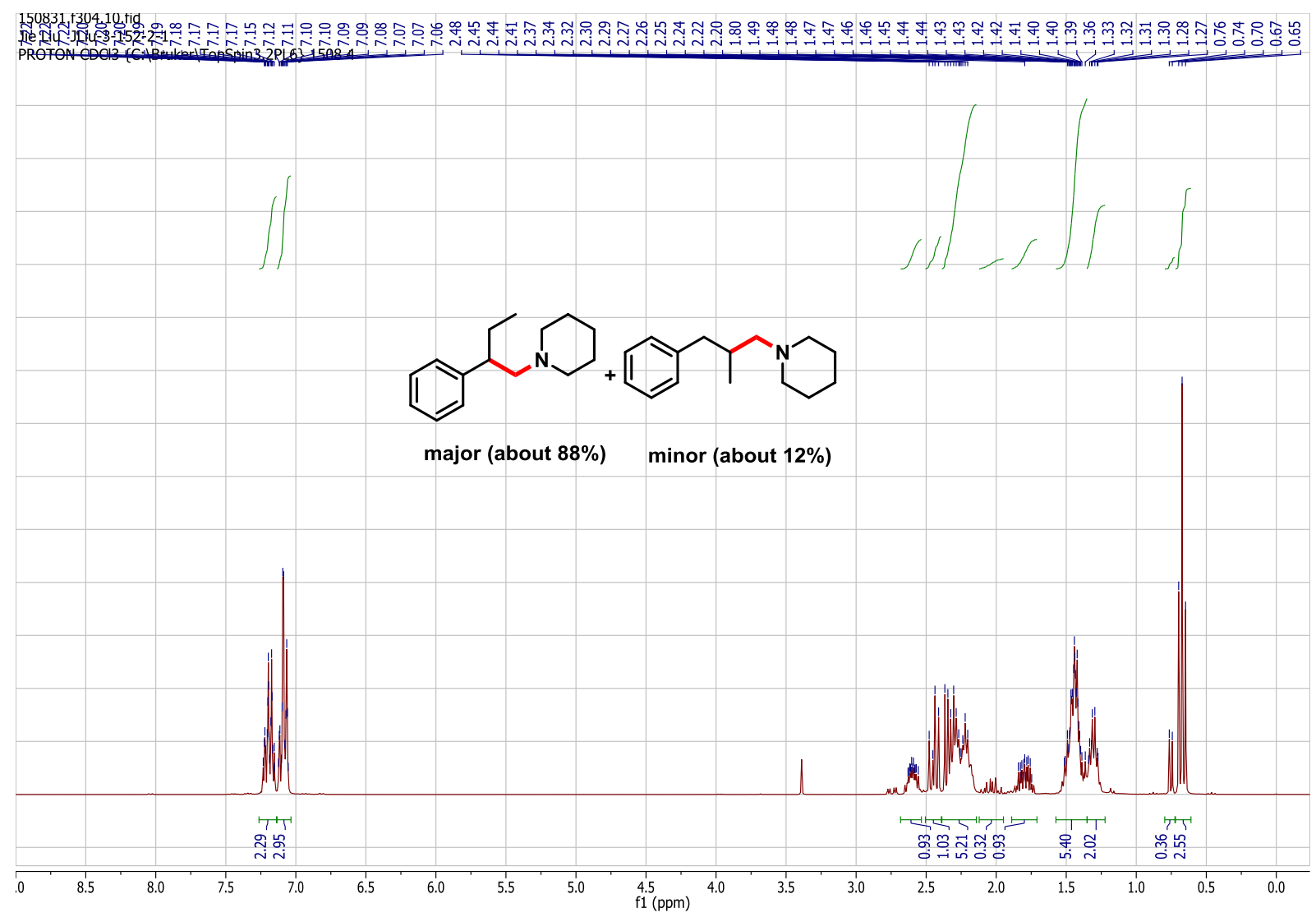

150831.f304.11.fid
Jie Liu JLiu-3-152-2-1

Jie Liu JLiu-3-152-2-1
C13CPD CDCl3 \{C:|Bruker|TopSpin3.2PL6\} 15084

CI3CPD CDCl3 \{C: Bruk

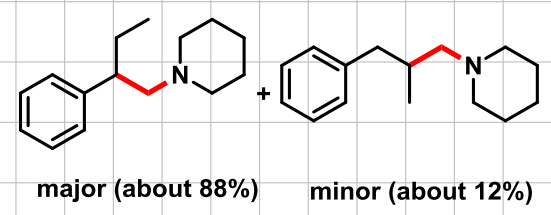

major (about $88 \%$ ) minor (about $12 \%$ )

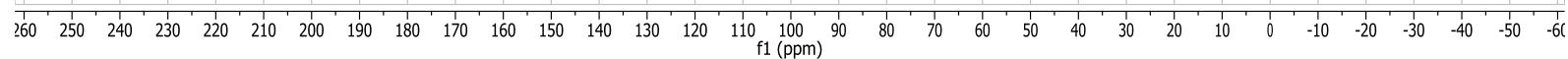



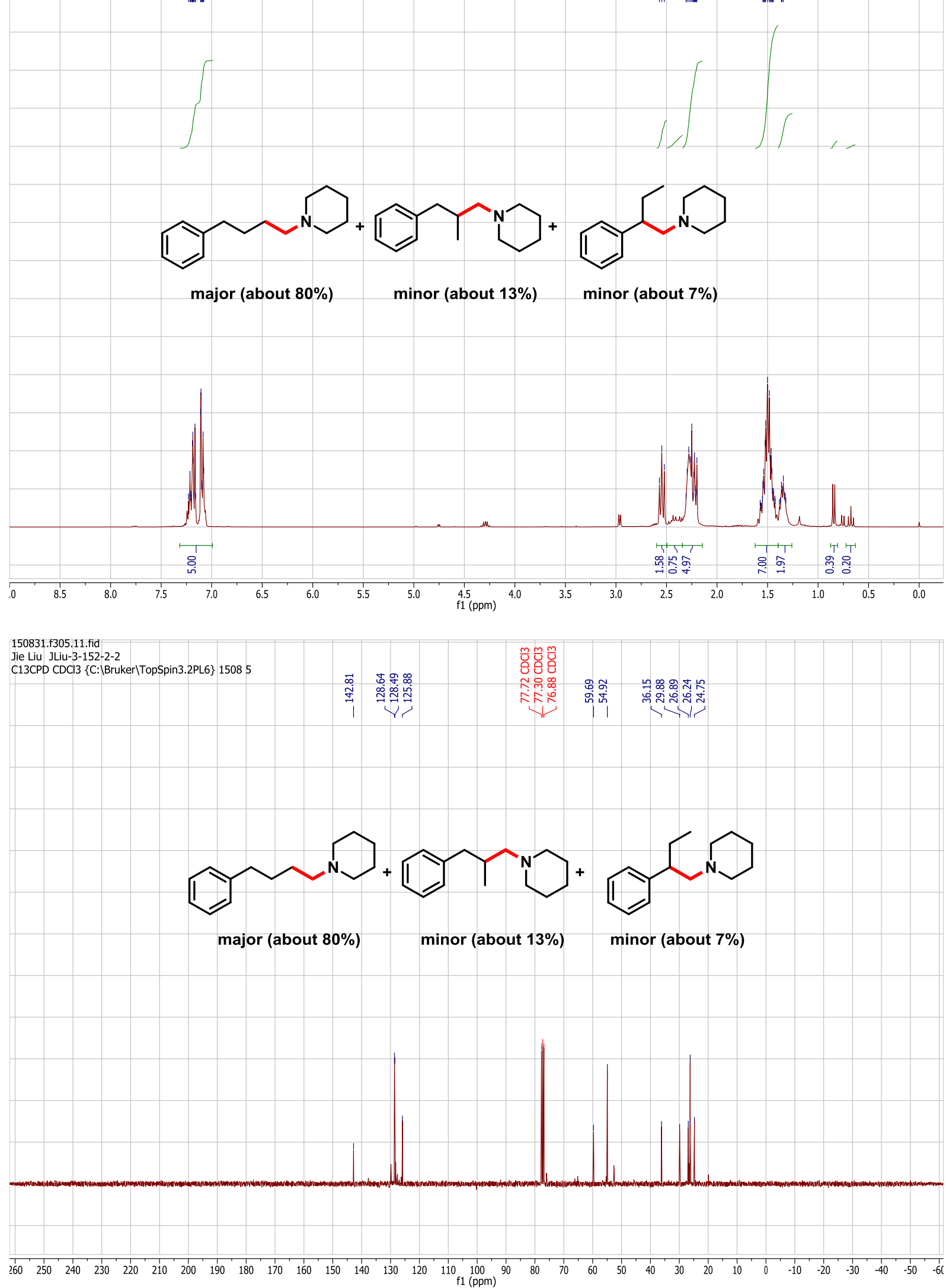

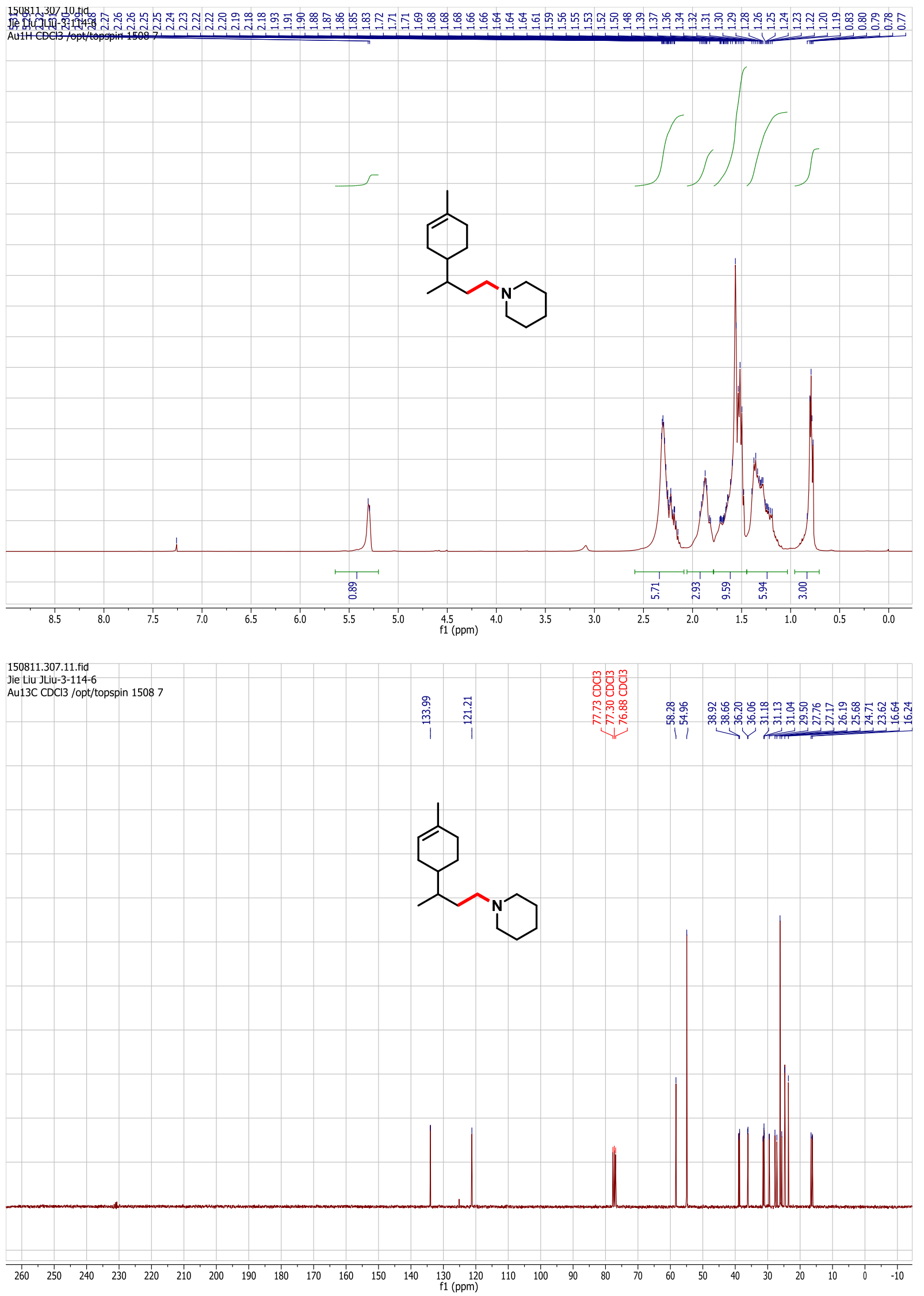

S26 
150814.302 Aull CDCl

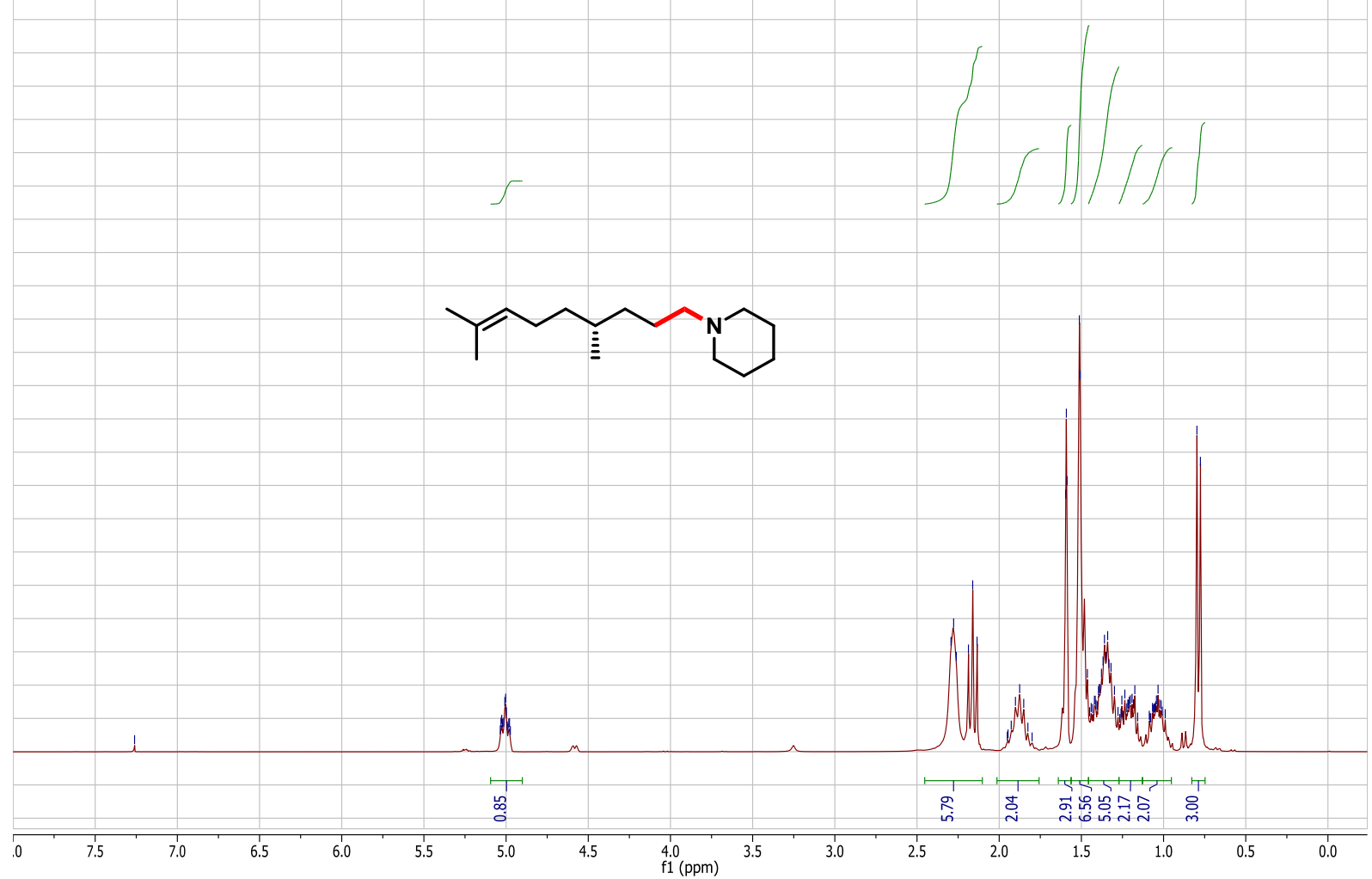

150814.302.11.fid

Ju13C CDCl3 /opt/topspin 15082

号

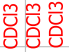

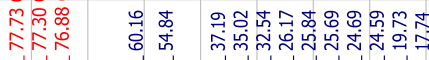

少 $\quad 1 \mid$
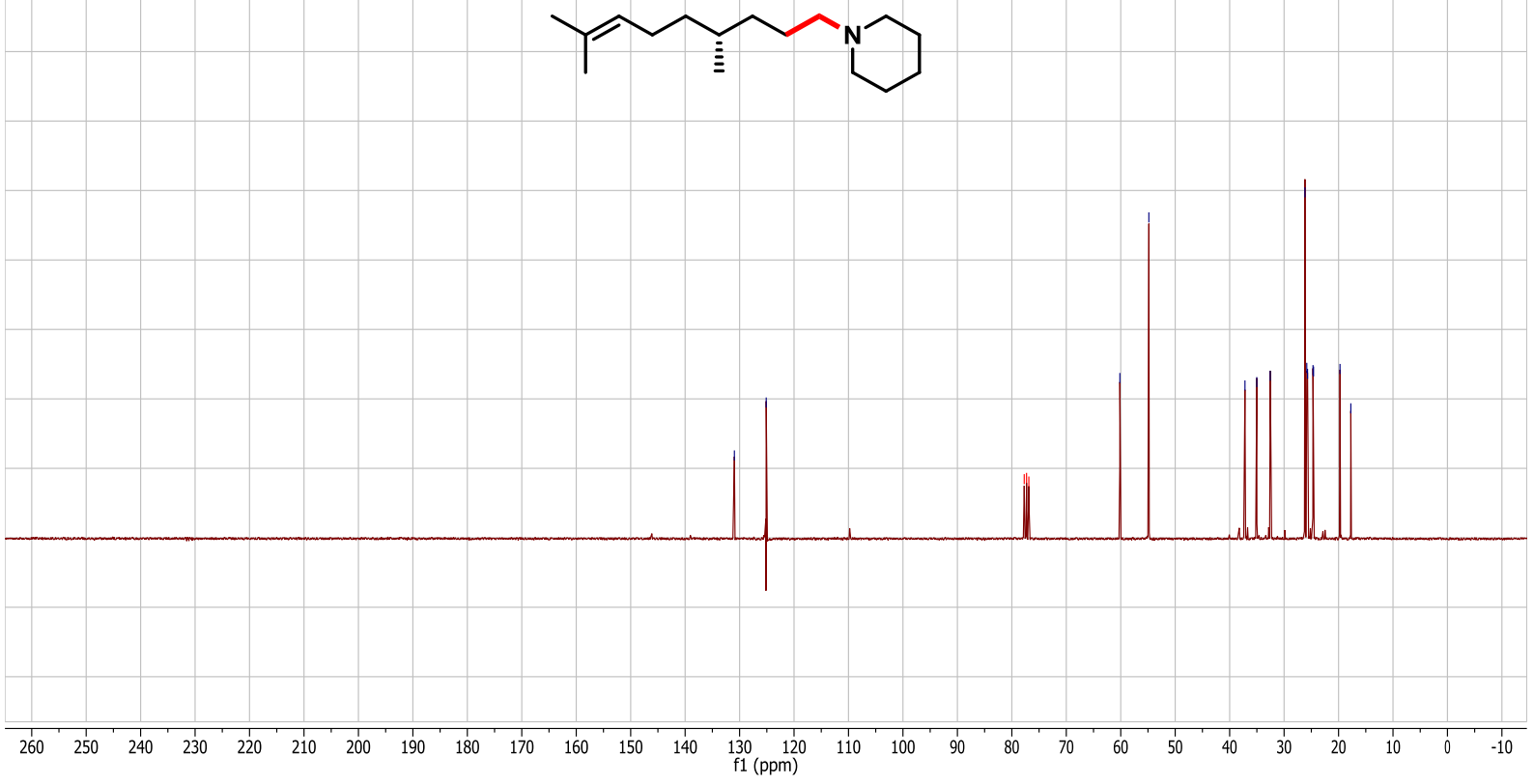

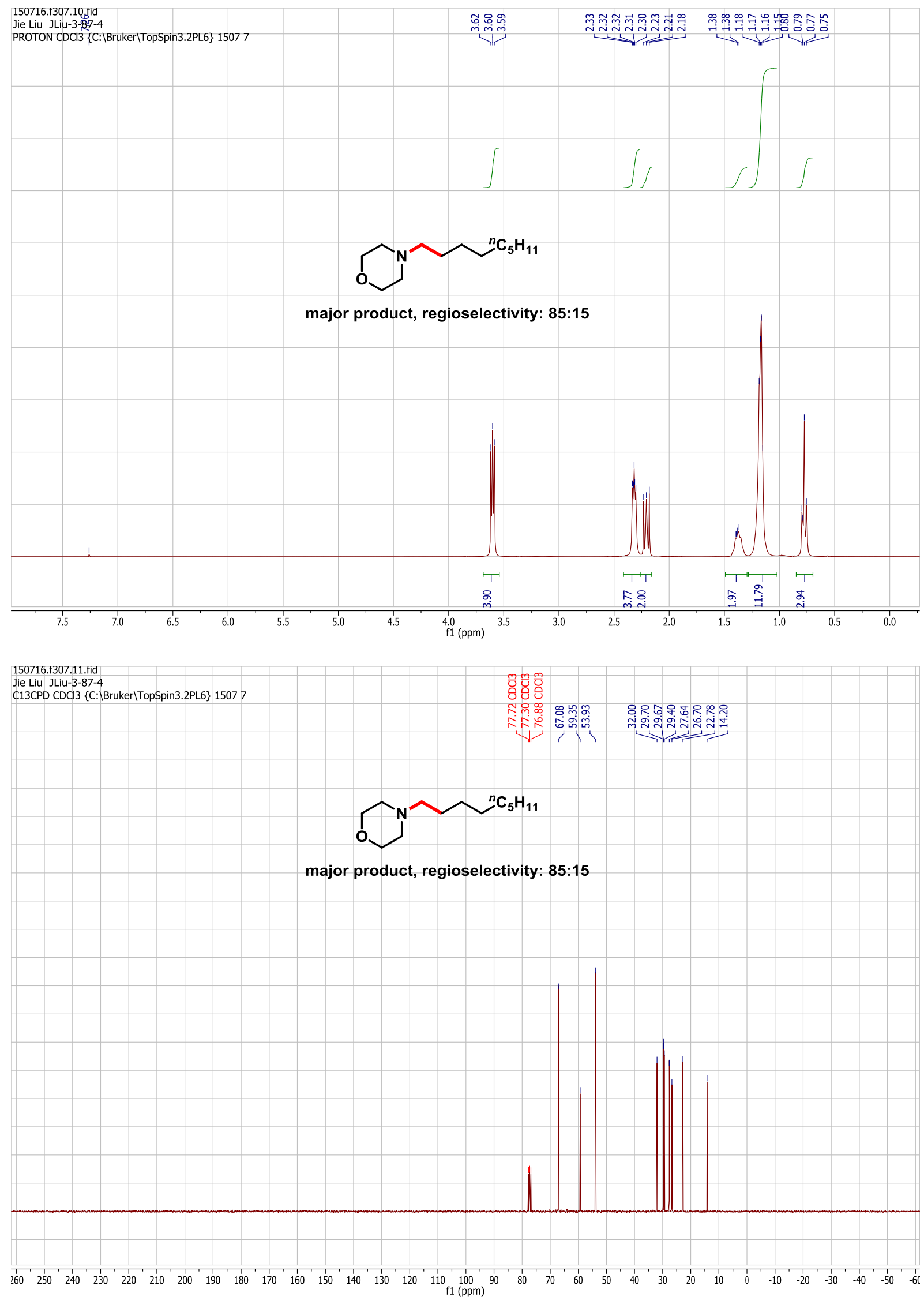

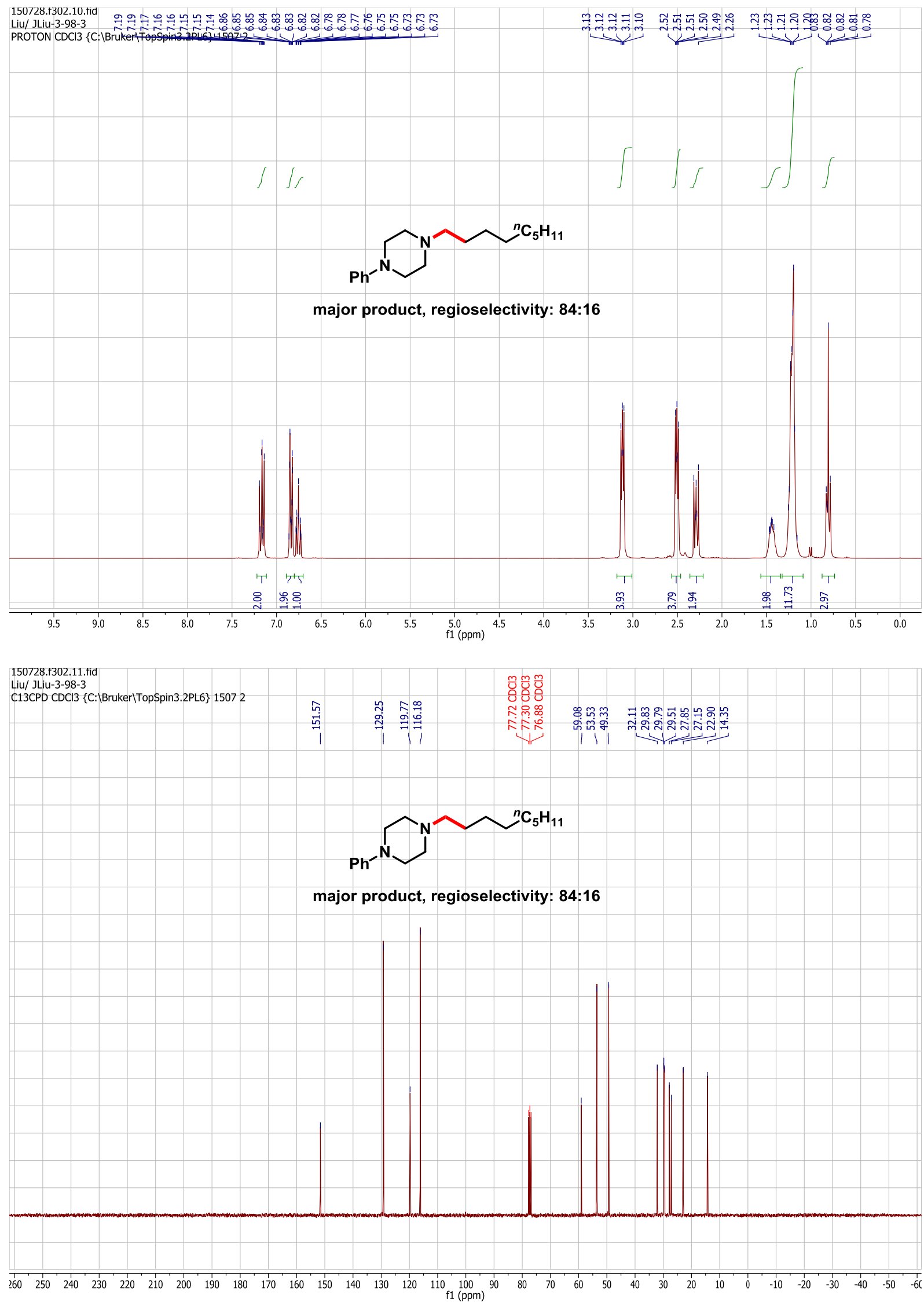


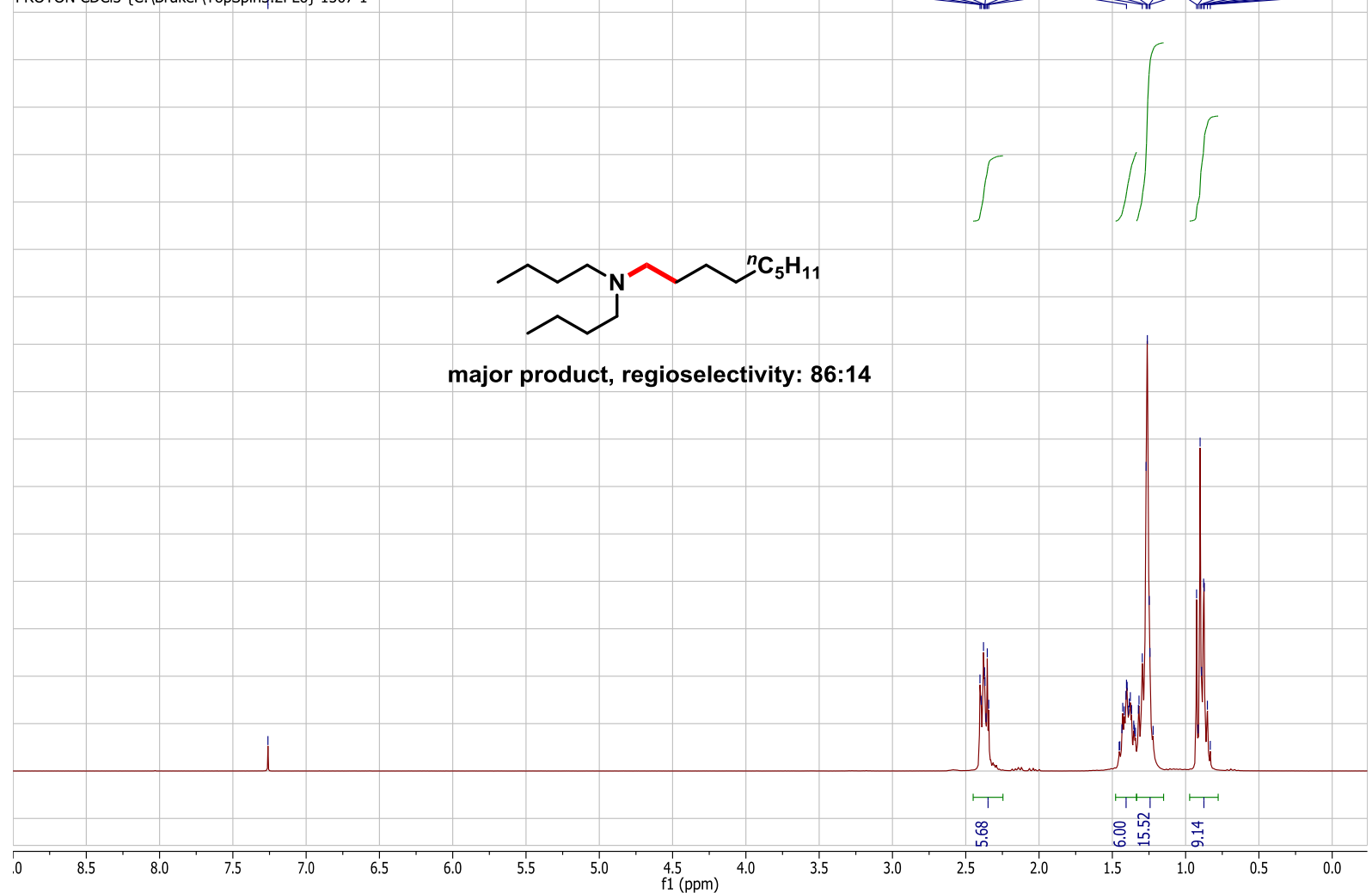

150806.f301.11.fid
Jie Liu JLiu-3-114-3

Jie Liu JLiu-3-114-3
C13CPD CDCI3 \{C:|Bruker|TopSpin3.2PL6\} 15071

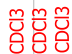

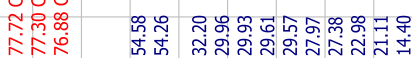

V

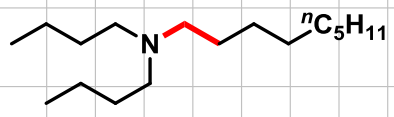

major product, regioselectivity: $86: 14$

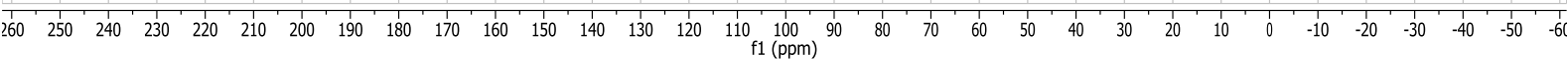




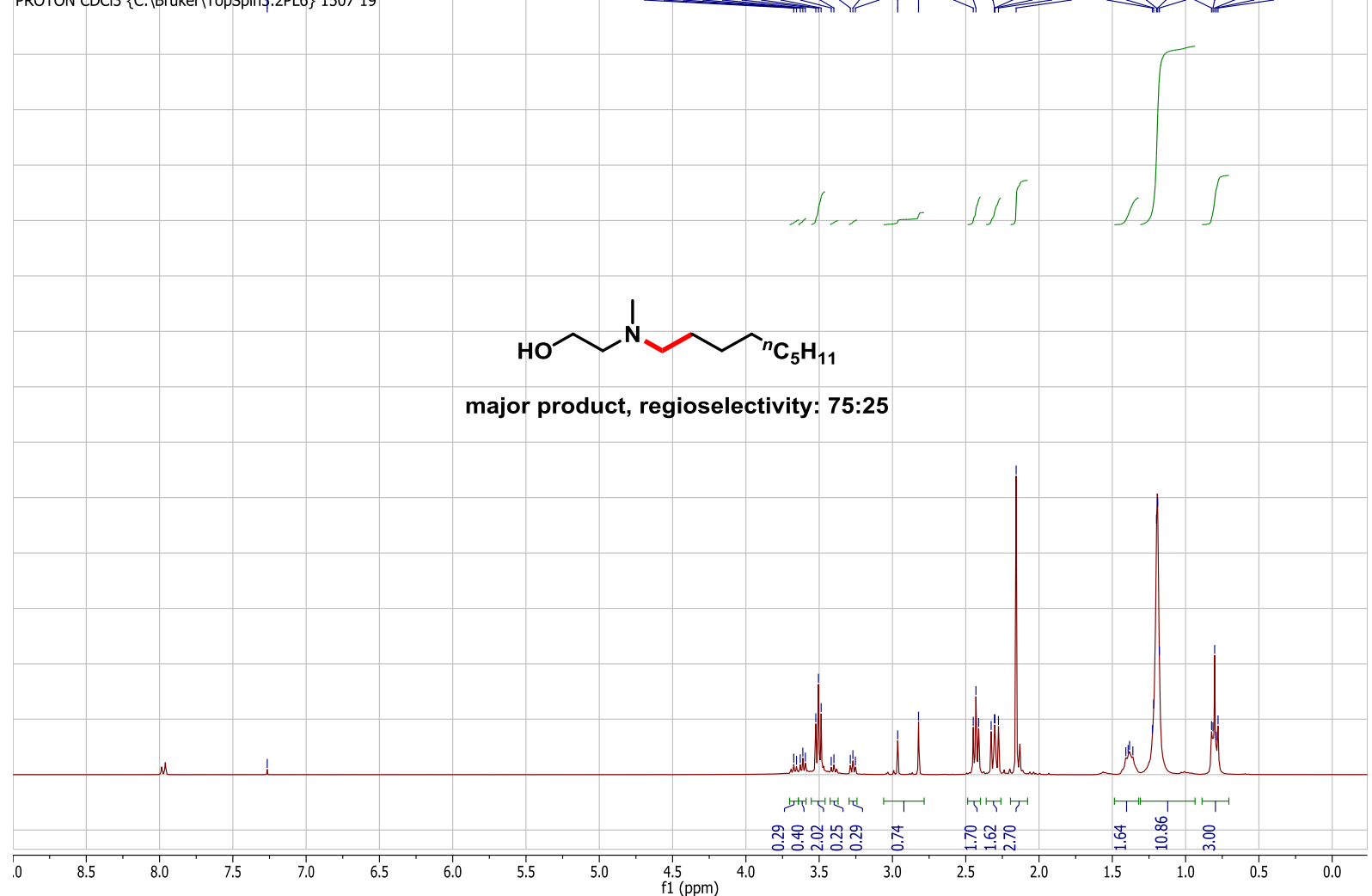

150722.f319.11.fid
Jie Liu JLiu-3-98-1-2

Jie Liu JLiu-3-98-1-2
C13CPD CDCl3 \{C:|Bruker|TopSpin3.2PL6\} 150719

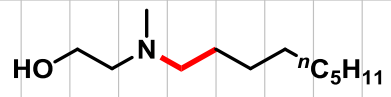

major product, regioselectivity: $75: 25$

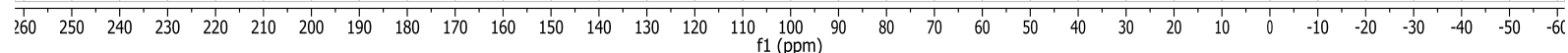



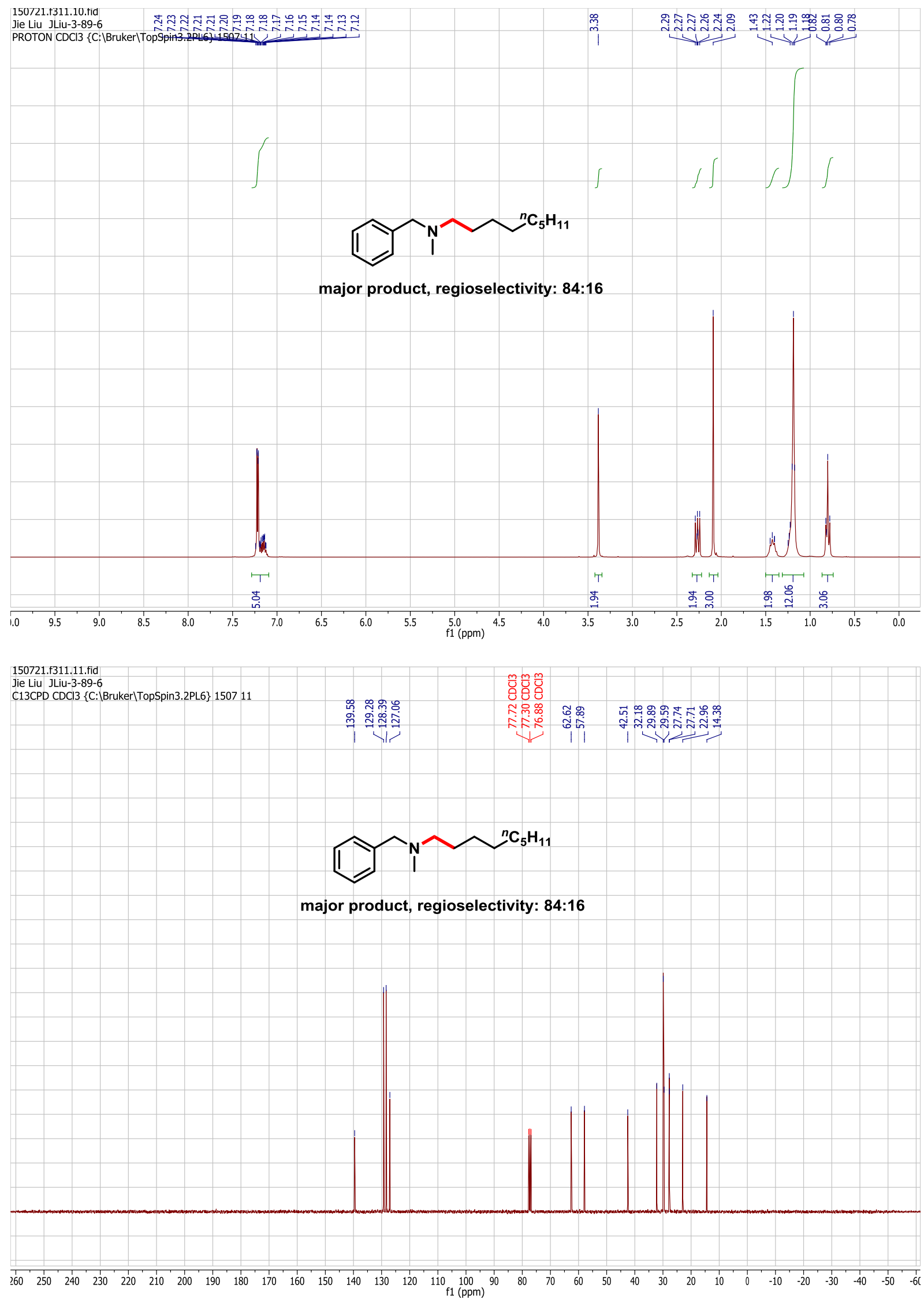

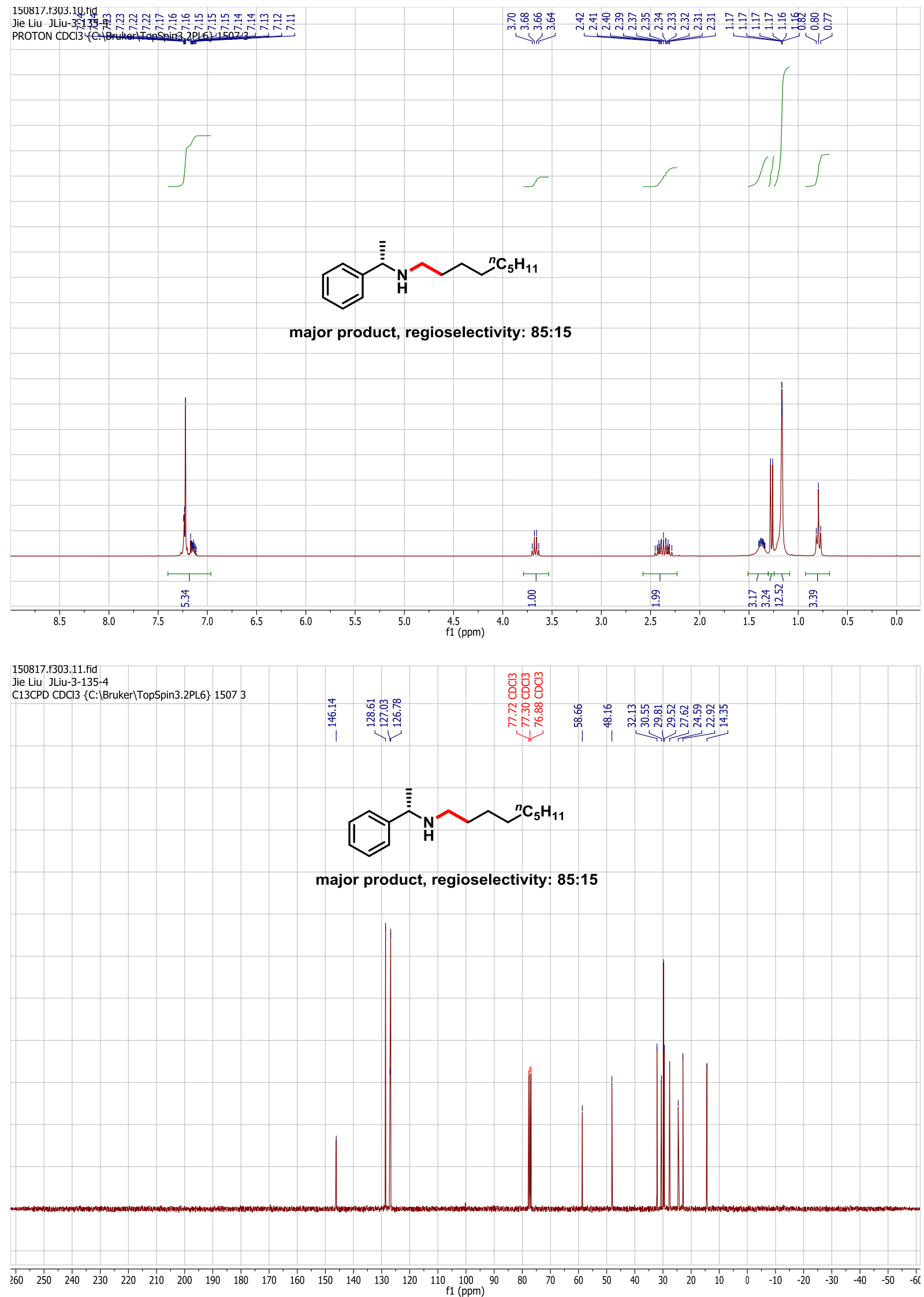


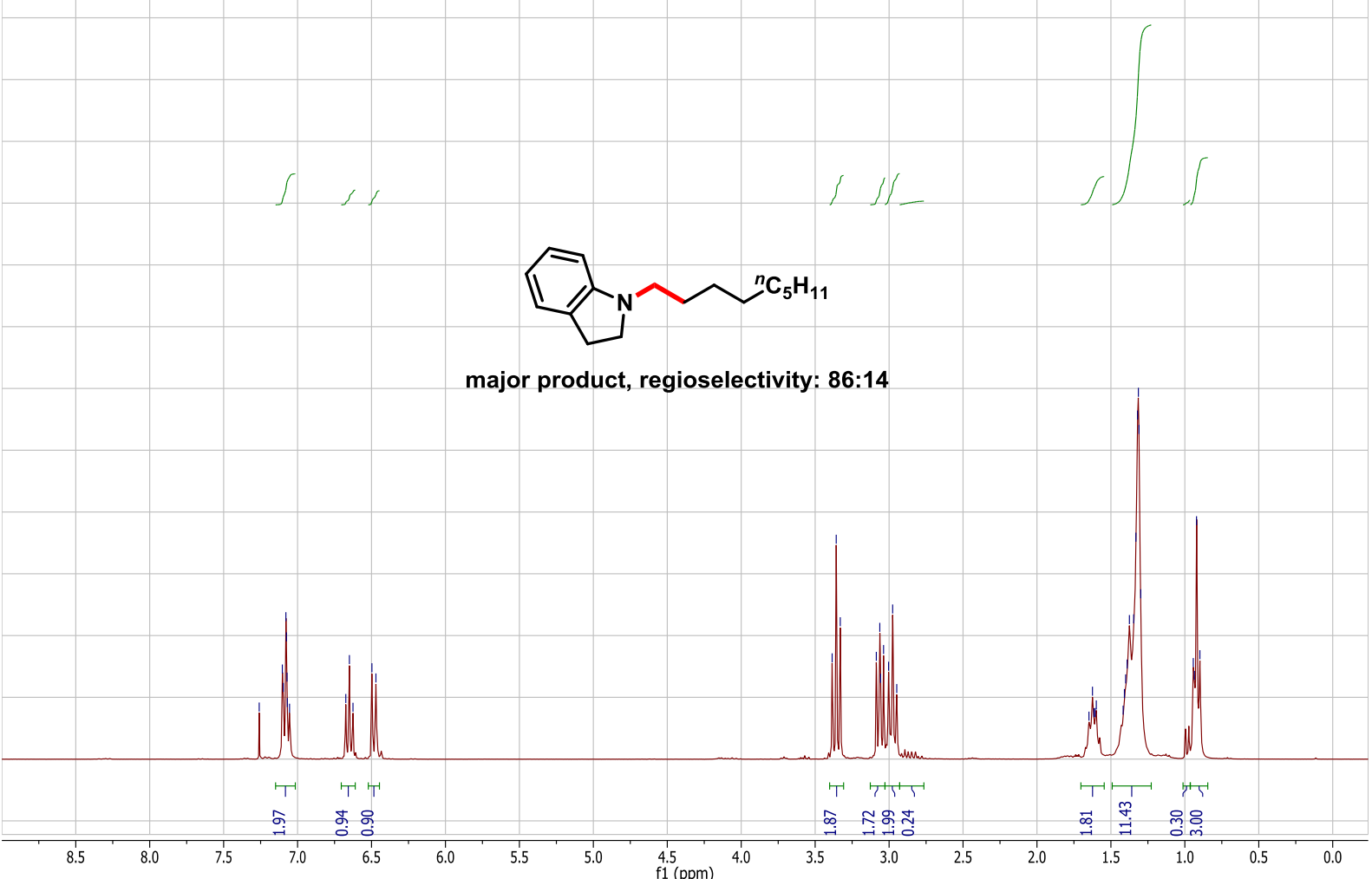

150723.f314.11.fid
Jie Liu JLiu-3-91-4

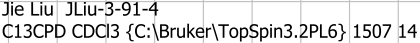
$\stackrel{t}{\circ}$

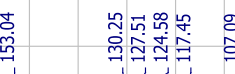

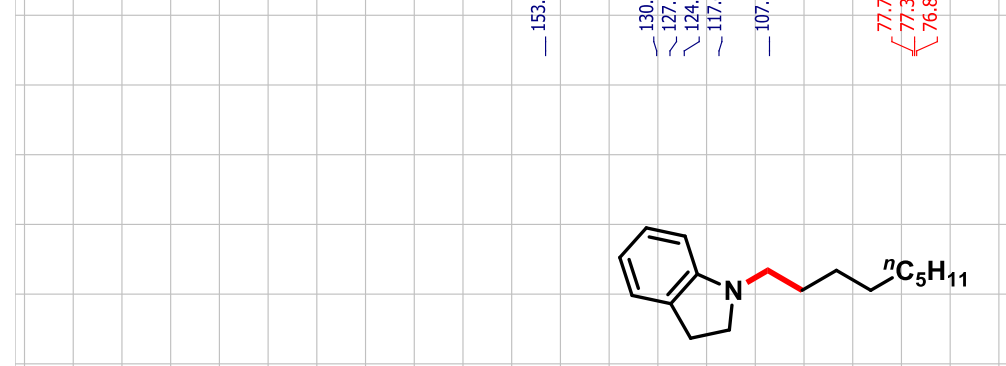

major product, regioselectivity: $86: 14$

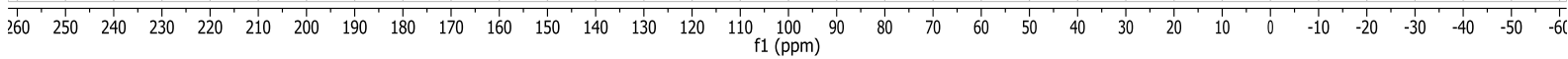




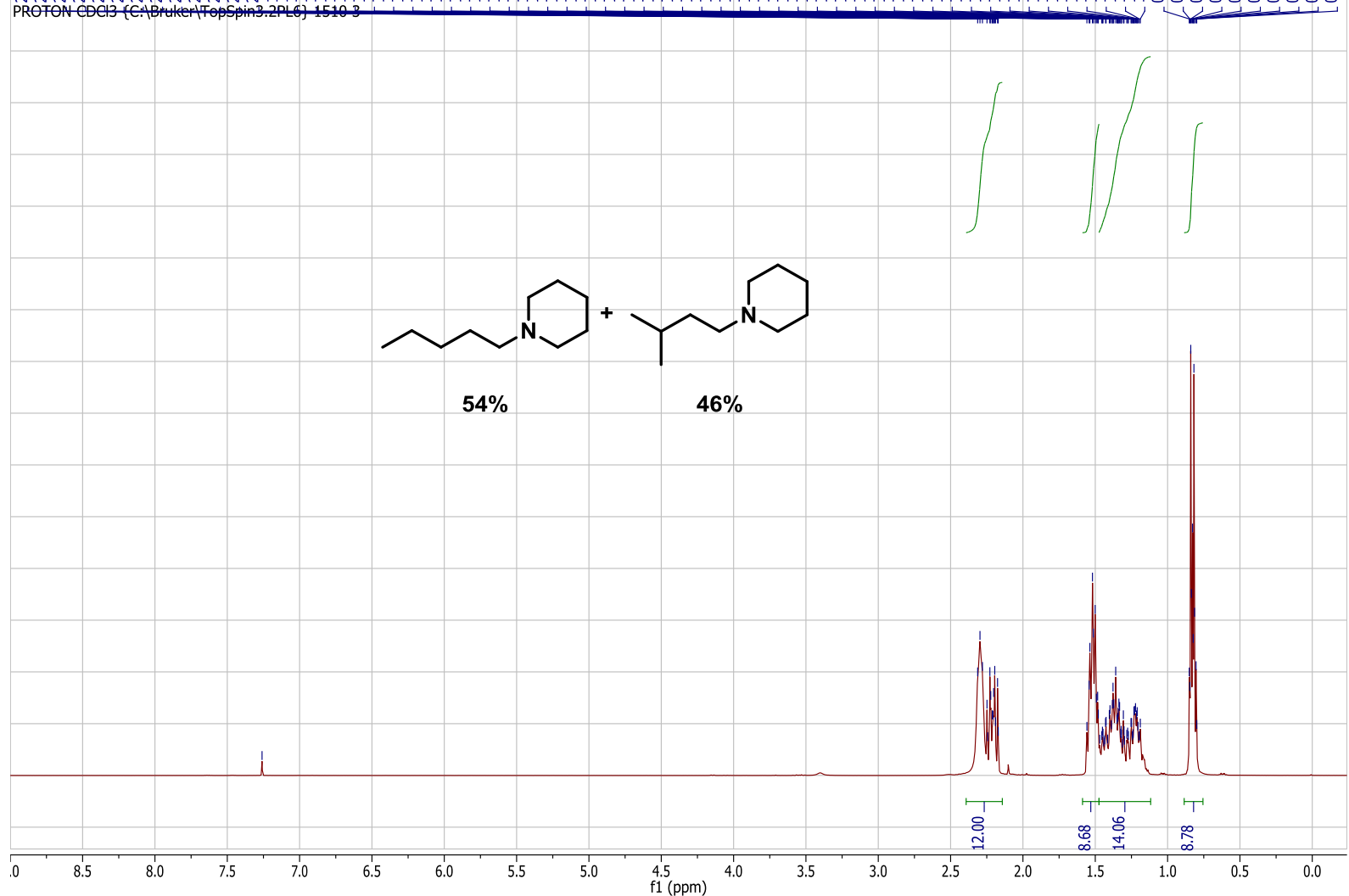

151012.f303.11.fid
Jie Liu JLiu-4-6

C13CPD CDCl3 \{C:|Bruker|TopSpin3.2PL6\} 15103

응몽뭉

상유 @

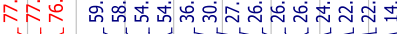

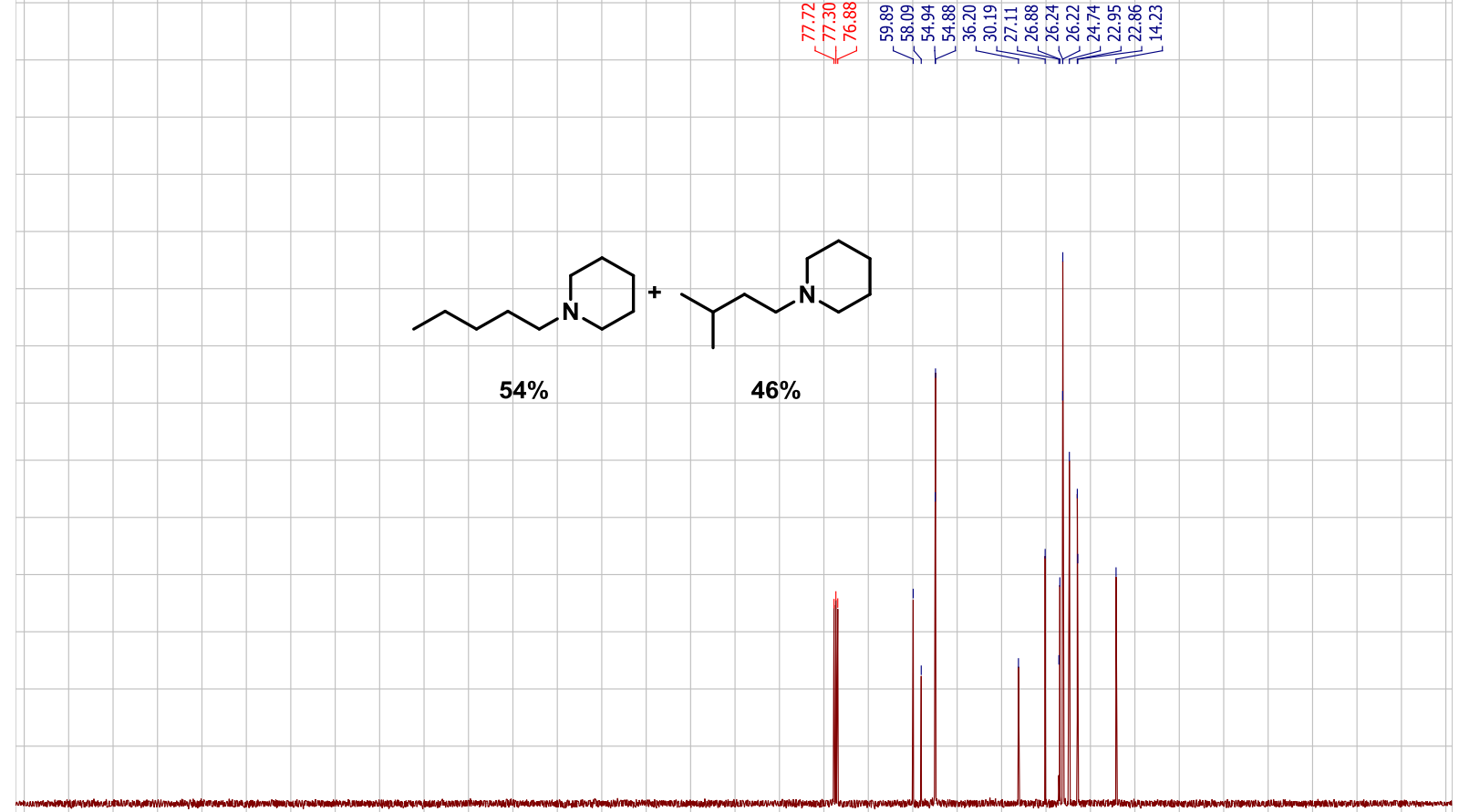

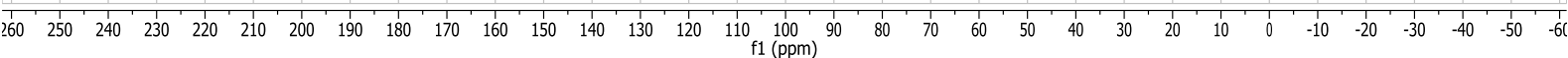

